NASA Technical Memorandum 85950

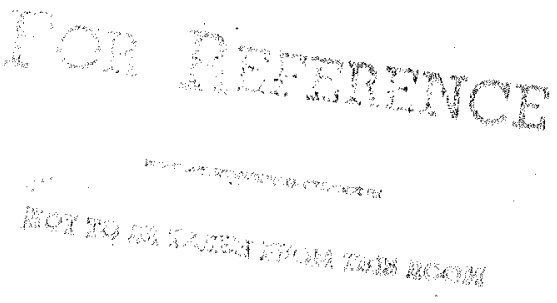

\title{
Numerical Optimization Design of Advanced Transonic Wing Configurations
}

\section{Gary B. Cosentino and Terry L. Holst}

May 1984

\section{LEMANY COPY

$$
191984
$$

MULLY RESEARCH CENTE 
NASA Technical Memorandum 85950

\section{Numerical Optimization Design of Advanced Transonic Wing Configurations}

Gary B. Cosentino, NASA Ames Post-Baccalaureate Program, Consortium Agreement, University of Colorado, Boulder, Colorado

Terry L. Holst, Ames Research Center, Moffett Field, California 
This Page Intentionally Left Blank 


\section{CONTENTS}

ABSTRACT $\ldots \ldots \ldots \ldots \ldots \ldots \ldots \ldots \ldots \ldots \ldots \ldots \ldots \ldots \ldots \ldots \ldots \ldots$

ACKNOWLEDGMENTS $\ldots \ldots \ldots \ldots \ldots \ldots \ldots \ldots \ldots \ldots \ldots \ldots \ldots \ldots \ldots$ vi

LIST OF TABLES $\ldots \ldots \ldots \ldots \ldots \ldots \ldots \ldots \ldots \ldots \ldots \ldots \ldots \ldots \ldots$ vii

LIST OF FIGURES $\ldots \ldots \ldots \ldots \ldots \ldots \ldots \ldots \ldots \ldots \ldots \ldots \ldots \ldots \ldots \ldots \ldots$

CHAPTER

I. INTRODUCTION $\ldots \ldots \ldots \ldots \ldots \ldots \ldots \ldots \ldots \ldots \ldots \ldots \ldots \ldots \ldots \ldots \ldots$

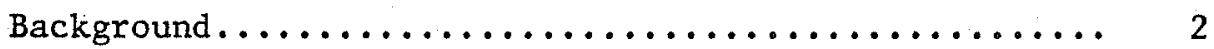

Transonic Computational Design Methods........... 5

Current Approach....................... 11

II. TRANSONIC WING FLOW-FIELD SOLUTION............. 15

Governing Equations..................... 15

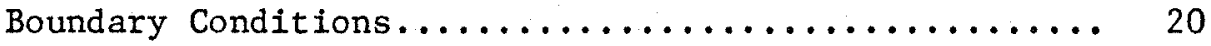

Grid Generation....................... 21

III. NUMERICAL OPTIMIZATION..................... 24

Gradient Computation.................... 27

The Quasi-Newton Method Optimization Iteration....... 28

Termination Criteria.................... 30

Finite-Difference Step-Size Selection............ 30

IV. AERODYNAMIC DESIGN PROGRAM DEVELOPMENT........... 33

Wing Geometry Generation.................. 35

Evaluation of Objective Function.............. 37

Geometrical Perturbation Technique.............. 39 
V. WING DESIGN CASE RESULTS................... 42

Lockheed C-141B Wing Design................ 43

Gates Learjet Century III Wing Design........... 57

Cessna Mode1 650 Wing Design.................. 68

VI. DISCUSSION AND CONCLUSIONS.................. 82

REFERENCES $\ldots \ldots \ldots \ldots \ldots \ldots \ldots \ldots \ldots \ldots \ldots \ldots \ldots \ldots \ldots \ldots \ldots \ldots \ldots \ldots \ldots \ldots$ 
Numerical Optimization Design of Advanced Transonic Wing Configurations

Gary B. Cosentino, University of Colorado

Terry Holst, NASA Ames Research Center

A computationally efficient and versatile technique for use in the design of advanced transonic wing configurations has been developed. A reliable and fast transonic wing flow-field analysis program, TWING, has been coupled with a modified quasi-Newton method, unconstrained optimization algorithm, QNMDIF, to create a new design tool. Fully three-dimensional wing designs utilizing both specified wing pressure distributions and drag-to-1ift ratio minimization as design objectives are demonstrated. Because of the high computational efficiency of each of the components of the design code, in particular the vectorization of TWING and the high speed of the Cray X-MP vector computer, the computer time required for a typical wing design is reduced by approximately an order of magnitude over previous methods. The shock-wave drag, a quantity computed by the wing flow-field analysis algorithm, was previously thought to be too unreliable to be used as the objective function to be minimized. In the results presented here, this computed wave drag has been used as the quantity to be optimized (minimized) with great success, yielding wing designs with nearly shock-free (zero wave drag) pressure distributions and very reasonable wing section shapes. The optimized wing configurations exhibit improved lift-todrag ratio performance characteristics. 


\section{ACKNOWLEDGMENTS}

The authors are greatly indebted to the following persons for their support, advice, and help throughout the course of this research: Prof. C.-Y. Chow of the University of Colorado, Mr. Raymond M. Hicks of NASA Ames Research Center, and Mr. Scott D. Thomas and Mr. Robert A. Kennelly of Informatics General Corporation. Their invaluable help and advice was very much appreciated. Special thanks go to Dean A. Richard Seebass of the University of Colorado for initiating the author's participation in this research project; to Dr. Helmut Sobieczky of the DFVLR, Gottingen, West Germany, for suggesting the spline support movement technique and providing the wing geometry generator; and finally to Mr. Richard J. Crupper of the Cessna Aircraft Company for providing the Cessna Mode1 650 wing geometry.

This research was conducted on the NASA Ames Cray X-MP Serial 103 supercomputer. Funds for the support of this study were allocated by the NASA Ames Research Center, Moffett Field, California, under Interchange No. NCA2-OR-170-202. 
vii

\section{TABLES}

Table

1. Summary of Lockheed C-141B Wing Design............ 48

2. Summary of Gates Learjet Century III Wing Design....... 66

3. Summary of Cessna Mode1 650 Wing Design............. 80 
FIGURES

Figure

1. Schematic of General $(x, y, z)$ to $(\xi, \eta, \zeta)$ transformation. (a) Physical domain; (b) computational domain..... 17

2. Two views of the Lockheed $\mathrm{C}-141 \mathrm{~B}$ wing geometry with aspect ratio 7.89 and leading-edge sweep of $27.7^{\circ}$.

(a) Planform view; (b) isometric view.............. 44

3. Location of the fixed $(+)$ and movable $(*)$ splinesupport points on the $G A(W)-2 M O D$ airfoll $\ldots \ldots \ldots \ldots \ldots . . .46$

4. TWING/QNM drag-to-lift ratio minimization result, Lockheed C-141B wing design: root span station........ 49

5. TWING/QNM drag-to-1ift ratio minimization result, Lockheed $\mathrm{C}-141 \mathrm{~B}$ wing design: $24.2 \%$ span station....... 50

6. TWING/QNM drag-to-lift ratio minimization result, Lockheed C-141B wing design: $48.5 \%$ span station....... 51

7. TWING/QNM drag-to-1ift ratio minimization result, Lockheed C-141B wing design: $72.7 \%$ span station....... 52

8. TWING/QNM drag-to-1ift ratio minimization result, Lockheed $\mathrm{C}-141 \mathrm{~B}$ wing design: $90.9 \%$ span station....... 53

9. Objective function $\left(C_{D} / C_{L} \times 100\right)$ versus the number of TWING flow solutions: $C-141 B$ wing.............. 55

10. Coefficient of drag $(\times 100)$ versus Mach number for the original and optimized wing $\ldots \ldots \ldots \ldots \ldots \ldots \ldots \ldots \ldots$

11. Two views of the Gates Learjet Century III wing geometry with aspect ratio 6.72 and leading-edge sweep of $15.9^{\circ}$. (a) Planform view; (b) isometric view....... 58

12. Location of the fixed (t) and movable (*) splinesupport points on the NACA $64_{1} A 212$ airfoil........... 59

13. TWING/QNM drag-to-lift ratio minimization result, Gates Learjet Century III wing: root span station...... 61

14. TWING/QNM drag-to-1ift ratio minimization result, Gates Learjet Century III wing: $24.2 \%$ span station..... 
15. TWING/QNM drag-to-1ift ratio minimization result, Gates Learjet Century III wing: $48.5 \%$ span station...... 63

16. TWING/QNM drag-to-lift ratio minimization result, Gates Learjet Century III wing: $72.7 \%$ span station..... 64

17. TWING/QNM drag-to-1ift ratio minimization result, Gates Learjet Century III wing: $90.9 \%$ span station..... 65

18. Objective function $\left(C_{D} / C_{L} \times 100\right)$ versus the number

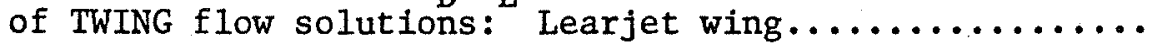

19. TWING/QNM pressure-design mode result, Gates Learjet Century III wing planform: root span station..........

20. TWING/QNM pressure-design mode result, Gates Learjet Century III wing planform: $48.5 \%$ span station........ 70

21. TWING/QNM pressure-design mode result, Gates Learjet Century III wing planform: $90.9 \%$ span station........ 71

22. Two views of the Cessna Citation III Mode1 650 wing geometry with aspect ratio 9.0 and leading-edge sweep of $27.2^{\circ}$. (a) Planform view; (b) isometric view.........

23. Location of the fixed ( + ) and movable (*) splinesupport points on the three Cessna airfoils. (a) Root station; (b) break station; (c) tip station............ 74

24. TWING/QNM drag-to-lift ratio minimization result, Cessna Model 650 wing design: root span station........ 75

25. TWING/QNM drag-to-1ift ratio minimization result, Cessna Mode1 650 wing design: $24.2 \%$ span station...... 76

26. TWING/QNM drag-to-1ift ratio minimization result, Cessna Model 650 wing design: $48.5 \%$ span station...... 77

27. TWING/QNM drag-to-1ift ratio minimization result, Cessna Model 650 wing design: $72.7 \%$ span station....... 78

28. TWING/QNM drag-to-1ift ratio minimization result, Cessna Model 650 wing design: $90.9 \%$ span station....... 79

29. Objective function $\left(C_{D} / C_{L} \times 100\right)$ versus the number

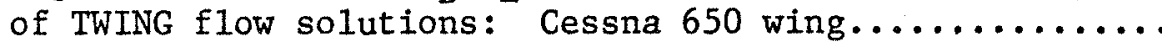


CHAPTER I

\section{INTRODUCTION}

The challenge of designing modern commercial transport and military fighter aircraft to be efficient and practical while also demanding higher performance levels is one of the most perplexing tasks facing the aerospace engineer. The next generation of commercial transports will be required to meet new standards of fuel efficiency and productivity that surpass those of even the most advanced aircraft in service today. Increases in productivity generally mean increases in the nominal cruise Mach number, which in turn make the transonic drag-rise effects more pronounced. Thus, new design techniques that will permit aircraft operation at higher speeds while minimizing adverse drag caused by compressibility effects will be important aids to the transport aircraft designer. Advanced fighter aircraft designs must meet a somewhat different yet equally challenging set of performance criteria. Fuel efficiency during nontactical cruise, high-speed maneuverability, and the requirement for operations in the subsonic, transonic, and supersonic flight regimes are seemingly conflicting objectives to be traded off by the designer. Additionally, other considerations are becoming increasingly important in the final design, such as radar cross-sectional area and weapons carrying capability. Clearly, a systematic method of measuring the effect of configuration changes 
on each of these design objectives is necessary in order to help the design engineer select an acceptable solution. Indeed, an automated system capable of finding the "best compromise" to a problem such as aircraft design involving several important and contradictory objectives would be very useful.

\section{Background}

Specific examples may be easily found among aircraft flying today which operate in one or all of the four possible flight regimes; namely, the subsonic, transonic, supersonic, and hypersonic regimes. Among these, it is the transonic flight regime that poses some of the most interesting problems for the designer, and this report addresses techniques applicable to this regime. Characteristic of this transonic regime are regions of mixed flow, or areas where subregions of subsonic, sonic, and supersonic flow velocities are in proximity. For free-stream Mach numbers near but less than unity, the disturbance created by the presence of some body will cause an acceleration of the flow in its vicinity such that regions of local supersonic flow will appear. The transition back to free-stream subsonic flow will generally be made nonisentropically through a shock wave.

As an example, consider a two-dimensional airfoil placed in a high subsonic free-stream flow. For a particular loading, or lift coefficient value, there will be one free-stream Mach number, the critical Mach number, at which sonic flow will first be achieved at some point on the airfoil surface. As the free-stream Mach number 
is increased, this sonic point will expand continuously into a dome of supersonic flow above the airfoil, and will usually be terminated by a shock wave. On a fully three-dimensional wing, this characteristic supersonic dome becomes a surface, extending outboard from the wing root, and decreasing in size toward the tip, where the loading (and hence the flow acceleration) is small. The shock wave then exists as a sheet along the span of the wing.

The phenomenon described above, also termed compressibility effects, defines the essence of the transonic-flow problem. Compressibility effects are responsible for a significant increase in drag as an aircraft operates at higher cruise Mach numbers in an effort to reduce time in flight and thereby increase productivity. In more severe cases, the appearance of strong shock waves near the trailing edge of a wing can induce control-surface oscillations known as "aileron buzz," which may have destructive effects. An interesting account of the history and difficulties associated with transonic aerodynamics is given by Spreiter [1].

Meeting the efficiency needs of the airline operators for the next generation of transports is a challenge that will require improved design methods, both for new aircraft designs and redesigns of existing transports. Several new approaches, including research on composite structural materials and the implementation of active control systems for aerodynamic load control and alleviation, are already being used. Both of these approaches aid in reducing an aircraft's weight, either by the use of lighter materials of construction, or by permitting a reduction in the required contro1surface areas. Decreases in engine specific fuel consumption can 
also be expected from the new generation of high-bypass-ratio turbofan engines. The Lockheed L-1011 trijet transport incorporates all these design advances.

An interesting subset of the new approaches available to an aircraft designer is that comprising aerodynamic design improvements in general, and design for optimum cruise performance in particular. A survey of this subject is given by Lynch [2]. Although performance improvements from purely aerodynamic modifications are small, even a $5 \%$ reduction in cruise drag on a DC-10-type aircraft may save $\$ 500,000$ per airplane per year in fuel costs [2]. For this reason, the use of new design techniques which offer improvements in this area is becoming increasingly attractive in the aerospace industry. Among the alternatives available to the aircraft designer is the digital computer, the performance of which has been dramatically increased in recent years. Supercomputers operating in a vectorprocessing mode are capable of executing many millions of floatingpoint calculations per second, and they are generally available to industries at reasonable costs. Complementing their power are improvements in new computational fluid dynamic algorithms which can make full use of the vector-processing abilities of these computers. The scope of the problems that may be addressed, as we11 as the accuracy of the final computed result, has been significantly increased in recent years. In addition, the cost of these computations has been reduced. Concurrently, the energy and labor costs associated with wind-tunnel testing have risen sharply, making the computational fluid dynamics tools even more attractive. 
This report deals with the development of an efficient new tool, which utilizes existing mathematical and computational fluid dynamics (CFD) algorithms, for use in the aerodynamic design of configurations operating in the transonic regime. Specifically, the objective is to develop a technique for computationally designing wing configurations that yield optimal transonic cruise performance and improved lift-to-drag ratio characteristics. Given a suitable starting configuration, the geometry of the wing is then systematically altered until the desired objectives are achieved. It should be noted that the applicability of a configuration so designed is limited only by the assumptions inherent in the CFD algorithm.

\section{Transonic Computational Design Methods}

An excellent and concise review of existing transonic design methods and their limitations is given by Holst et a1. [3]. A brief outline of these methods, their uses, points of merit, and limitations will be presented in the following paragraphs. Complete descriptions of the technical aspects of these methods and their implementation can be found in the cited references.

There are essentially three different categories of numerical design techniques available to the design engineer for use in the aerodynamic tailoring of airfoil and wing shapes. These are the inverse methods, the indirect methods, and the direct methods. Each offers a different way of attacking the same basic problem of finding the aerodynamic shape for efficient operation in the 
transonic (or other) flight regime, without resorting to expensive cut-and-try wind-tunnel testing.

Inverse methods derive their name from the fact that they attempt to solve the problem backwards. That is, given a desired flow field (and hence pressure distribution) about some body, the problem is to find the unknown body geometry. An immediate advantage here is that the designer can specify all of the aerodynamic forces and their distributions acting on the configuration, such as lift, drag, and pitching moment, through proper specification of the pressure field. Thus, if a wing surface can be found that will yield the specified pressure distribution, it should automatically satisfy all requirements and constraints. The major disadvantage is that the designer, after having specified the pressure field, has no control over the surface shape defined by the inverse code. The problem is highly nonlinear, and it is difficult to eliminate unacceptable surface shapes by making small remedial changes in the specified pressures. Additionally, mathematical non-uniqueness in both the inverse problem formulation and the potential-flow solution may complicate the procedure further. These effects are even more pronounced in three dimensions [3]. Indirect design methods are characterized by the fact that the designer has little or no control over the final configuration or the aerodynamics produced by it. Only an initial or baseline geometry is specified, and the indirect code introduces modifications to this geometry such that its objectives, for example, shock-free flow, are achieved. Usually, some parameters require adjustment by 
the user for each particular design case; however, it is usually the case that these parameters have nonphysical interpretations. Thus, in the absence of extensive user experience, trial-and-error may be necessary to establish the correct value of these parameters for a particular application. The hodograph method (in two dimensions) as first used by Nieuwland [4], and the fictitious-gas technique (in two and three dimensions) of Sobieczky [5] and Sobieczky et al. [6] are examples of indirect design methods. Because the fictitious-gas technique is not limited to two-dimensional design, as is the hodograph method (both seek configurations which yield shock-free flow), it will be briefly described.

The equations governing flow in the transonic regime are of three different types determined in terms of the local Mach number. The flow in the subsonic regions is governed by an elliptic equation, yielding inherently smooth and continuous solution data. On the sonic line, or the surface defining the boundary of the supersonic dome, the Mach number is unity, and the governing equation is parabolic. Inside this sonic surface, the flow is supersonic, and the equation type is hyperbolic. Unlike the smooth nature of the solutions to the subsonic elliptic equations, the solutions to a hyperbolic equation allow for the existence of jumps or other discontinuities, which are interpreted physically as shock waves. In the elegant technique of Sobieczky, an artificial or fictitious gas law is brought into play whenever the local Mach number exceeds 1 . Thus, regions of hyperbolic behavior are reverted to smooth elliptic behavior, producing data on the sonic line corresponding to a 
shock-free (or discontinuity-free) flow. With this important data on the sonic line used as an initial condition, the method of characteristics is used to find a stream surface that continues the original airfoil (or wing) surface into the supersonic region. If this marching is successful, a shock-free design is found. If a limit-1ine intervenes during the marching, it indicates that no physical solution supporting shock-free flow is possible under the current conditions. Here one may either change the flow conditions, alter the initial geometry, or re-adjust parameters that control the fictitious-gas law used.

The procedure described above has been shown to work well in the presence of viscous-inviscid interactions in two-dimensional designs [7] and three-dimensional designs [8] using existing computational codes. It has been successfully applied to a practical airfoil design problem by Cosentino [9] and to several wing designs by Fung et a1. [10], Raj et al. [11], and Yu [12]. Its main limitation seems to be that it is strictly a point-design method; that is, the shock-free performance can be expected only from operations very close to the design conditions. Although off-design performance should not suffer severely, shocks will again reappear at both slightly higher and lower Mach numbers. Further, the differences in the computed and actual viscous effects may be sufficient to preclude the realization of shock-free transonic flow in actual flight conditions.

The third category of design procedures, direct design methods, is generally made up of those procedures involving design by numerical optimization. Here, a computational flow-field 
analysis program is coupled with a numerical optimization algorithm in such a way as to create a design tool. This procedure will be the main topic of this report.

The aerodynamic quantities such as lift, drag, and pitching moment are computed by the CFD algorithm for a certain configuration and are used in defining an objective function to be minimized by the optimizer. Thus, this objective function must relate improvements in the aerodynamic quality of the design to a decrease in function value. Minimization of this objective function, through proper choice of the pertinent geometric design variables, should then correspond to a configuration that is "optimal" in some sense. Insofar as aerodynamic design is concerned, it is clear that a configuration yielding a minimum drag count, while still satisfying reasonable design constraints (such as wing thickness, planform size and shape, and twist variations), should be an optimal configuration design. Although the above is true only for a given flight condition, it is possible to find a design that will satisfy (as nearly as possible) these optimal requirements for a range of flight regimes by the use of multiple design points. It is in fact this ability of numerical optimization techniques to solve multiple design point problems that makes their use attractive.

An example of the need for this "best compromise design" strategy is the helicopter rotor design problem. Here, there are essentially two distinct operating conditions in which the rotor blade must perform. The advancing blade sees both the forward speed of the helicopter and its own rotational speed as the total freestream speed. The retreating blade, conversely, sees a velocity 
corresponding to the difference between the rotational speed and the forward vehicle speed. There is also a difference in angle of attack in general. Both conditions prevail half of the time, thus both are equally important design points. Clearly, a rotor design that is a best compromise between these two conditions would be desired.

In addition to the merits of multiple-point designs, numerical optimization design (NOD) procedures also allow the designer a great deal of control over both the aerodynamic qualities and the physical shape of the final configuration design. However, a great deal of user expertise may be necessary in order to take advantage of this high degree of flexibility. It is, at least, available, which is generally not true of the inverse or indirect design methods. By far the most persistent criticism of NOD procedures is the large amount of computer time required for the optimization algorithm to "sort out" and decide which configuration is best. However, this is largely time spent by the CFD algorithm in computing the flow. With the rapid improvements in computer and algorithm speed that are taking place today, this shortcoming may soon be largely eliminated. An excellent survey of past work in this field is given by Hicks [13], who points out some of its successes and failures. A case study on the Lockheed C-141B military transport is given by Lores and Hinson [14], and an application to Learjet-type airfoil design is presented by Hinson [15] . 


\section{Current Approach}

The main purpose of this work is to expand the technique of transonic configuration design by numerical optimization and to demonstrate that most of its shortcomings can be alleviated. This is accomplished primarily by bringing several new technological advances into play in a new design program. Among these new technologies are advances in algorithm speed for computing the wing flow field, more intelligent optimization routines, and the great increase in computing power afforded by supercomputers such as the Cray X-MP. Some discussion of these advances will be given in the following paragraphs.

The transonic wing analysis code used in this work is the very fast and reliable TWING (transonic wing) program developed at NASA Ames Research Center by Holst and Thomas [16]. It solves the transonic full-potential equation for the inviscid, irrotational compressible flow past an isolated wing on a wall. TWING utilizes the fully implicit approximate-factorization (AF2) algorithm [17] for solving the full-potential equation. This algorithm displays rapid convergence and robustness for a wide range of flow-field cases. In addition, it permits the computer code to be written in a form that will allow vector processing on a vector computer, greatly increasing the speed of execution [18]. Running on the Cray X-MP, TWING provides well converged flow-field solutions in as little as 10 to $20 \mathrm{sec}$ for typical cases.

Most of the work done in the area of numerical optimization design of aerodynamic configurations has utilized the CONMIN 
(constrained-function-minimization) algorithm of Vanderplaats [19]. In the new approach of the present study, an alternative method, known as the quasi-Newton method of unconstrained optimization, is used. In a recent comparative study by Kennelly [20], the optimization code QNMDIF (quasi-Newton method with difference approximations to the derivatives) of Gill et a1. [21] and Gill and Murray [22] was shown to be more efficient and to perform better than CONMIN. Another innovative feature of the present work is the manner in which geometrical perturbations are introduced into the wing shape. Previously, a number of terms based on sine or exponential "bump" functions were assembled in a Fourier-like series and added to a surface of the airfoil section or wing. A criticism of the use of such shape functions stems from the fact that they have no known physical basis. In an interesting new approach by Aidala et al. [23], special "aerofunction shapes" are created by using a twodimensional inverse code. Specific local changes in a pressure distribution are made, and the corresponding shape modification introduced to an airfoil is extracted and normalized to create a new series of shape functions. Because each shape function corresponds to a distinct (and desirable) pressure change, these functions may be thought of as being orthogonal in some sense. Thus, better efficiency should be expected from a numerical optimization design program using these new shape functions. In three dimensions, however, the creation of these special shape functions requires a more complex approach [23], and possibly a substantial computational effort. 
In the present work, a more straightforward technique of geometry perturbation is applied: the coordinate points defining the airfoil geometry are moved directly by the optimizer, then a spline curve is fitted through these points to produce a new shape. The number and location of these movable points are at the discretion of the designer, and a careful choice will result in a smooth and physically reasonable shape being defined for each iteration. This new procedure is enhanced by the use of a versatile geometry generation routine based on a program developed by Sobieczky [24] Aerodynamic drag, as computed by the flow-analysis program, would appear a natural choice for the objective function to be minimized by the optimizer. However, little success with this approach, particularly in three-dimensional design, is reported in the literature. Non-uniqueness effects may be present in the problem, and the pressure distribution about a configuration designed by a drag minimization approach can be physically unreasonable [3]. Another criticism is that the accuracy, or numerically generated "noise" inherent in the computed drag value relays incorrect gradient information to the optimization routine. A study of this phenomenon revealed that with the advanced algorithms in use in the new design program, sufficient accuracy appeared to be present in the computed drag to warrant a reexamination of this design objective. If successful, this would represent the first time that useful threedimensional aerodynamic designs were obtained by the technique of drag minimization. 
The performance increases that may be obtained by combining these technologies in a new design program might well redefine the range of applicability for this design method. The large increase in performance, combined with the great flexibility of the technique, should make the use of NOD procedures very attractive to the aerospace commity. 
CHAPTER II

\section{TRANSONIC WING FLOW-FIELD SOLUTION}

The construction of any numerical optimization design program for use in aerodynamic applications begins with the analysis program itself. This program provides the aerodynamic analysis for each new modified configuration during the design process. From the pressure field that is computed for the configuration, the aerodynamic force coefficients are derived. This information, regarding the quality of a particular design, is related to the optimizer via the objective function. The accuracy and precision of this objective is critical to the success of the design program, for changes to a design are made by the optimizer based on this information.

\section{Governing Equations}

The governing equation solved in the TWING analysis program is the three-dimensional, full-potential equation. This equation is written in strong conservation-law form, and is given by

$$
\left(\rho \phi_{x}\right)_{x}+\left(\rho \phi_{y}\right)_{y}+\left(\rho \phi_{z}\right)_{z}=0
$$

where

$$
p=\left[1-\frac{\gamma-1}{\gamma+1}\left(\phi_{x}^{2}+\phi_{y}^{2}+\phi_{z}^{2}\right)\right]^{1 /(\gamma-1)}
$$

The density $\rho$ and the velocity components $\phi_{x}, \phi_{y}$, and $\phi_{z}$ are nondimensionalized by the stagnation density $\rho_{s}$ and the critical 
speed of sound $a^{*}$, respectively. The coordinate system used here is the $x, y$, and $z$ Cartesian system in the streamwise, spanwise, and the vertical directions, respectively. The parameter $\gamma=\mathrm{C}_{\mathrm{p}} / \mathrm{C}_{\mathrm{v}}$ is the ratio of specific heats.

The assumptions inherent in the application of this equation are that the flow is steady, isentropic, irrotational, and hence, inviscid. These are quite reasonable assumptions for an aircraft in cruise flight at transonic Mach numbers at which only weak shock waves persist, and at which the boundary layer remains attached to the surface. Although shock waves represent a source of energy dissipation and, therefore, entropy production, this effect is negligible if the shocks are acceptably weak (local normal Mach number just ahead of the shock less than 1.3). This is generally the case on a well designed wing. If shocks exist that do not satisfy this condition they are always captured in a pessimistic fashion. That is, they are stronger than they should be. Having this situation built into the conservative potential formulation allows intermediate designs involving strong shocks. These solutions are just as valid as any other, providing the final design consists of (at most) a weak shock.

Next, by applying a general independent variable coordinate transformation to Equation (1), the physical (Cartesian coordinate) domain is transformed into the computational domain (Fig. 1). This transformation maintains strong conservation form and is given by

$$
\xi=\xi(\mathrm{x}, \mathrm{y}, \mathrm{z}) \quad \eta=n(\mathrm{x}, \mathrm{y}, \mathrm{z}) \quad \zeta=\zeta(\mathrm{x}, \mathrm{y}, \mathrm{z})
$$




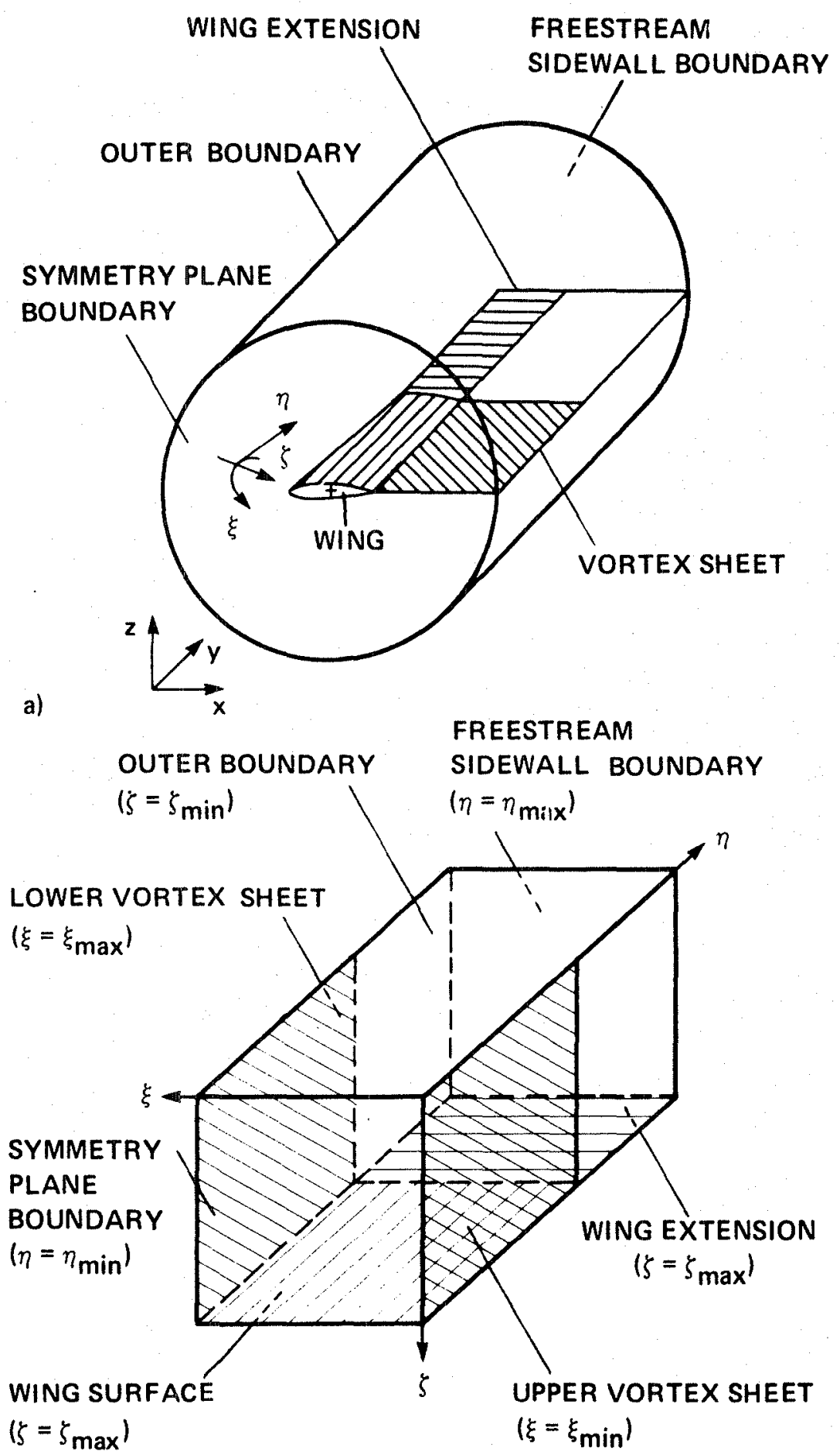

b)

Figure 1. Schematic of general $(x, y, z)$ to $(\xi, n, \zeta)$ transformation. (a) Physical domain; (b) computational domain. 
The full-potential equation written now in the computational domain $(\xi, \eta, \zeta$ coordinate system) is given by

$$
(\rho U / J)_{\xi}+(\rho V / J)_{\eta}+(\rho W / J)_{\zeta}=0
$$

and

$$
\rho=\left[1-\frac{\gamma-1}{\gamma+1}\left(U \phi_{\xi}+V \phi_{\eta}+W \phi_{\zeta}\right)\right]^{1 /(\gamma-1)}
$$

where the quantities $U, V$, and $W$ are the contravariant velocity

components along the $\xi, \eta$, and $\zeta$ directions, respectively, and are given by

$$
\begin{aligned}
& \mathrm{U}=\mathrm{A}_{1} \phi_{\xi}+\mathrm{A}_{4} \phi_{\eta}+\mathrm{A}_{5} \phi_{\zeta} \\
& \mathrm{V}=\mathrm{A}_{4} \phi_{\xi}+\mathrm{A}_{2} \phi_{n}+\mathrm{A}_{6} \phi_{\zeta} \\
& \mathrm{W}=\mathrm{A}_{5} \phi_{\xi}+\mathrm{A}_{6} \phi_{n}+\mathrm{A}_{3} \phi_{\zeta}
\end{aligned}
$$

The six quantities $A_{1}$ through $A_{6}$ are the metrical quantities of the transformation and are computed as

$$
\begin{aligned}
& A_{1}=\xi_{x}^{2}+\xi_{y}^{2}+\xi_{z}^{2} \\
& A_{2}=\eta_{x}^{2}+\eta_{y}^{2}+\eta_{z}^{2} \\
& A_{3}=\zeta_{x}^{2}+\zeta_{y}^{2}+\zeta_{z}^{2} \\
& A_{4}=\xi_{x} \eta_{x}+\xi_{y} \eta_{y}+\xi_{z} \eta_{z} \\
& A_{5}=\xi_{x} \zeta_{x}+\xi_{y} \zeta_{y}+\xi_{z} \zeta_{z} \\
& A_{6}=\eta_{x} \zeta_{x}+\eta_{y} \zeta_{y}+\eta_{z} \zeta_{z}
\end{aligned}
$$

The Jacobian of the transformation $\mathrm{J}$ is given by

$$
\begin{aligned}
& J=\xi_{x} \eta_{y} \zeta z+\xi_{z} n_{x}{ }^{\zeta}+\xi_{y} n_{z} \zeta x-\xi_{z} n_{y} \zeta x-\xi_{y} n_{x} z_{z}-\xi_{x} n_{z}{ }^{\zeta} \\
& =1 /\left(\mathrm{x}_{\xi} \mathrm{y}_{\eta} \mathrm{z}_{\zeta}+\mathrm{x}_{\zeta} \mathrm{y}_{\xi} \mathrm{z}_{\eta}+\mathrm{x}_{\eta} \mathrm{y}_{\zeta} \mathrm{z}_{\xi}-\mathrm{x}_{\xi} \mathrm{y}_{\zeta} \mathrm{z}_{\eta}-\mathrm{x}_{\eta} \mathrm{y}_{\xi} \mathrm{z}_{\zeta}-\mathrm{x}_{\zeta} \mathrm{y}_{\eta} \mathrm{z}_{\xi}\right)
\end{aligned}
$$


The following metric identities are required to evaluate the metric expressions of (5) and (6) above:

$$
\begin{aligned}
& \xi_{x}=J\left(y_{\eta} z_{\zeta}-y_{\zeta} z_{\eta}\right) \\
& \xi_{y}=J\left(x_{\zeta} z_{\eta}-x_{\eta} z_{\zeta}\right) \\
& \xi_{z}=J\left(x_{\eta} y_{\zeta}-x_{\zeta} y_{\eta}\right) \\
& \eta_{x}=J\left(y_{\zeta} z_{\xi}-y_{\xi^{z}} z_{\zeta}\right) \\
& \eta_{y}=J\left(x_{\xi^{2}}{ }_{\zeta}-x_{\zeta} z_{\xi}\right) \\
& n_{z}=J\left(x_{\zeta} y_{\xi}-x_{\xi^{y}} y_{\zeta}\right) \\
& \zeta_{x}=J\left(y_{\xi^{z}}{ }-y_{\eta} z_{\xi}\right) \\
& \zeta_{y}=J\left(x_{\eta} z_{\xi}-x_{\xi^{z}} z_{n}\right) \\
& \zeta_{z}=J\left(x_{\xi} y_{\eta}-x_{\eta} y_{\xi}\right)
\end{aligned}
$$

The metric quantities above [(Eqs. (5), (6), and (7)] are computed numerically by replacing all derivatives by the appropriate finitedifference formulas. These metrics are computed once for each new configuration supplied to TWING, and the flow-field solution then proceeds iteratively by solution of the governing equation.

The governing full-potential equation $[(3 a)$ and $(3 b)]$ is discretized using a spatial differencing formula suitable for solution by application of the AF2 iteration scheme. The fully implicit nature of this AF2 scheme allows for the rapid convergence of the solution to small residuals. That is, the first two orders of residual reduction take about as many iterations as do the second two orders. This is not the case with classical successive line overrelaxation (SLOR) schemes, which slow down dramatically as the 
residual drops. Thus, implementing an NOD technique where tight convergence is required with an AF2-based analysis scheme is a big advantage. Also, the AF2 algorithm has been written such that the required operations will take place in a vector processing mode on a vector computer such as the Cray X-MP. For these reasons, we11converged solutions (maximum level of residual at termination less than $0.5 \mathrm{E}-06$ ) are obtained for most cases in less than $20 \mathrm{sec}$. The details concerning the spatial differencing scheme formulas and the AF2 solution procedure will not be presented here; instead, the reader is directed to References 16 and 17 for further information. Details concerning the vectorization of the AF2 procedure are presented by Thomas and Holst in Reference 18 .

\section{Boundary Conditions}

At the wing-surface boundary of the flow, the condition of flow tangency is imposed; hence, it is required that there be no transpiration through the wing surface. This implies that the $\zeta$-contravariant velocity component be zero at the wing surface, or $\mathrm{W}=0$. This is specified numerically by the condition

$$
(\rho \mathrm{W} / \mathrm{J})_{i, j, N K+1 / 2}=-(\rho \mathrm{W} / J)_{i, j, N K-1 / 2}
$$

where $k=N K$ is the wing surface. In expressions where $\phi_{\zeta}$ is needed at the wing surface, the above $W=0$ boundary condition can be used again to obtain

$$
\left.\phi_{\zeta}\right|_{\text {wing }}=-\left(\mathrm{A}_{5} / \mathrm{A}_{3}\right) \phi_{\xi}-\left(\mathrm{A}_{6} / \mathrm{A}_{3}\right) \phi_{\eta}
$$


The final boundary condition that will be shown here is the important condition imposed at the symmetry plane or, physically, the wall on which the wing is mounted. Again, no transpiration is permitted through the wall (i.e., flow tangency is required and $\mathrm{V}=0$ ). This is accomplished by requiring that

$$
(\rho V / J)_{i, 1 / 2, k}=-(\rho V / J)_{i, 3 / 2, k}
$$

at $\mathbf{j}=1$, corresponding to the symmetry plane boundary.

Additional important boundary conditions imposed on the velocity potential are needed at the outer boundary and also at the free-stream sidewall boundary. Here, the initial free-stream distribution of potential is fixed for nonlifting cases. For lifting wings, the outer boundary is updated as the solution progresses by the usual compressible vortex solution with circulation $\Gamma_{j}[16]$.

\section{Grid Generation}

Since the geometry treated by TWING is the relatively simple case of an isolated wing on a flat wall, a simple two-dimensional grid-generation scheme is used at each span station. The extension to three dimensions is made by linear interpolation. A finitedifference mesh is fitted about each of the airfoil sections which make up the wing by solving the two Laplace equations

$$
\xi_{x x}+\xi_{z z}=0 ; \quad \zeta_{x x}+\zeta_{z z}=0
$$

at each defining station. These equations are solved in the computational domain as is the full-potential equation. The solution of Equations (11) is accomplished after differencing by employing the 
wel1-known ADI (alternating-direction-implicit) scheme. Then, with the $x$ and $z$ coordinates of the mesh at the defining stations known, the $y$ values along the span are obtained by using linear interpolation, subject to the planform shape specification. This simple method is capable of treating general wing geometries with arbitrary twist, taper, sweep, and dihedral, and yet is very efficient. Using $89 \times 25 \times 18$ grid points in the wraparound, spanwise, and radial directions, respectively, a mesh consisting of 40,050 total computational points may be generated in under $1 \mathrm{sec}$ of Cray X-MP CPU time. In an iterative design application (such as NOD) for which a new grid may be required for each new configuration, a fast grid-generation system helps considerably to reduce the overall computing time needed for the design. Further details concerning the grid generation used in TWING may be found in References 16 and 25 .

From the potential field obtained from the AF2 solution algorithm, TWING next computes the pressure distribution about the wing configuration. These pressures are then integrated to yield the overall forces acting on the wing. This pressure distribution, and the lift, drag, and pitching-moment coefficients are the output quantities of primary use in constructing an NOD program. By specifying the required accuracy of the computed potential solution (i.e., level of maximum residual before termination), the numerical "noise" level inherent in these output quantities can be controlled. Because of the speed of TWING relative to other similar programs, it 
is possible to specify much higher levels of convergence without incurring severe computational time penalties. Thus, greater levels of precision in the computed aerodynamic quantities may be achieved; this is vital to the success of the optimization program operating in the highly nonlinear realm of transonic flow. By far the most difficult quantity to utilize effectively as an optimization objective is the computed drag, primarily because of the low degree of precision associated with its computation. With TWING, it may be expected that the precision level of the drag coefficient will be greater than what was attainable in the past using slower algorithms. This is in fact largely the key to the success of the present method in obtaining useful wing designs by using computed drag in the objective function. 


\section{CHAPTER III}

\section{NUMERICAL OPTIMIZATION}

The term numerical optimization is generally used to describe a procedure by which the extrema of a function of $N$ variables are located with the aid of a digital computer. There is usually only one scalar function value involved and a onedimensional vector of length $\mathrm{N}$, corresponding to the $\mathrm{N}$ variables of the function. Depending on the particular numerical optimization algorithm used, the class of function that may be treated will vary. For the application of this work, namely transonic configuration design, the function (called the "objective function") can be expected to be highly nonlinear and perhaps occasionally discontinuous. In fact, it is well known that the appearance and disappearance of a shock wave are discontinuous phenomena. The characteristics of an objective function relating information concerning transonic flow demand an optimization algorithm capable of circumventing such highly nonlinear and discontinuous behavior; fortunately, they do exist.

The present work will employ a robust and relatively "intelligent" algorithm known as the quasi-Newton method which utilizes finite-difference approximations to the function derivatives, or QNMDIF for short. It is not a new technique, but its applications 
have not included transonic aerodynamic design until quite recently, when it was successfully integrated with a two-dimensional airfoil analysis program by Kennelly [20]. In that study, its performance was compared to that of the CONMIN algorithm [19], which has been used almost exclusively in transonic applications in the past. The new program QNMDIF was shown to be much more efficient insofar as the number of function evaluations needed to establish the location of the minimum is concerned. Additionally, QNMDIF should possess a better ability to cope with occasional functional discontinuities. Thus, it appears that a higher probability of success with a reduced computational effort is afforded by using QNMDIF.

As a smart algorithm, QNMDIF is able to build up curvature information, or in essence "learn" about the function it is trying to minimize. This is accomplished by constructing and periodically updating an approximation to the Hessian matrix, or matrix of mixed second partial derivatives of the objective function. This is in complement with the gradient vector, or one-dimensional array of first derivatives, which are evaluated using either forward or central finite-difference formulas. Because the accumulation of such curvature information is obtained at the expense of repeated function evaluations (which in the case of transonic flow-field solutions are not trivial), it is important that this information not be lost if it is necessary to restart a design calculation. Here, QMMDIF displays superior abilities relative to its predecessor CONMIN, for it was designed and modified with a restart capability in mind. 
The fundamental statement of the optimization problem may be formulated as

$$
\underset{x_{i} \in \mathscr{R}}{\operatorname{minimize}} F\left(x_{i}\right), \quad i=1,2, \ldots, N
$$

subject to

$$
\begin{array}{ll}
c_{i}\left(x_{i}\right)=0, & i=1,2, \ldots ., m \\
c_{i}\left(x_{i}\right) \leq 0, & i=m+1, \ldots, N
\end{array}
$$

Here, $F$ is the objective function of $N$ variables, and the $c_{i}$ 's are the constraint functions to be imposed on the solution. Taken together, these are termed the "problem functions" [26].

Whether constraints may be imposed on the solution is a characteristic of the optimization algorithm used. Such constraints preclude the possibility of the optimization program terminating upon locating a function minimum in an unacceptable sector of the design space. Generally, however, this usually slows the progress of the optimizer in locating any solution. For this reason, it is usually preferable to formulate the problem in such a way as to reduce or eliminate the need for constraints, and to utilize an algorithm intended for unconstrained optimization. QNMDIF is an example of a program designed for unconstrained function minimization, as opposed to CONMIN which may accept a number of constraints. After any constraints are eliminated, the optimization problem is reformulated simply as

$$
\underset{x_{i} \in \mathscr{R}}{\operatorname{minimize}} F\left(x_{i}\right), \quad i=1,2, \ldots, N
$$


This is the basic form of the problem that will be addressed in the following paragraphs concerning some basic concepts within the theory of numerical optimization. Extensive treatments may be found on many aspects of optimization theory in Reference 26 , and on the quasi-Newton method in particular in Reference 21.

\section{Gradient Computation}

In an application in which only objective function values are available and in which there is no possibility of obtaining a priori information about the nature of its derivatives, gradient information can only be obtained by using finite-difference approximations. The finite-difference intervals $h_{i}, i=1,2, \ldots ., N$, for differencing the objective function $F(x)$ with respect to each of the $N$ variables, are selected. The problem of selecting these intervals will be addressed later. With this $h$ vector determined, gradients may next be evaluated using standard forward- or centraldifference formulas, as

$$
g_{i}\left(x_{i}\right)=\left[F\left(x_{i}+h_{i} e_{i}\right)-F\left(x_{i}\right)\right] / h_{i}, \quad i=1,2, \ldots, N
$$

for forward differences, and

$$
g_{i}\left(x_{i}\right)=\left[F\left(x_{i}+h_{i} e_{i}\right)-F\left(x_{i}-h_{i} e_{i}\right)\right] / 2 h_{i}, \quad i=1,2, \ldots . N
$$

for evaluating central differences, where $e_{i}$ is the ith column of the identity matrix. Generally, the difference intervals $h_{i}$ are not altered during the optimization process. Since forward differencing will require only $N$ function evaluations per gradient 
computation, it is usually used first. If changes in the function value over the interval appear to be small compared with the relative precision in the function, the switch to central differences (requiring 2N evaluations per gradient estimation) will be made by the algorithm.

\section{The Quasi-Newton Method Optimization Iteration}

Having computed the objective function gradients as shown above, the actual optimization steps may begin. The quasi-Newton method attempts to locate the minimum of the objective by taking steps at each iteration $k$ in a direction specified by the curvature information previously acquired. Specifically, the search direction $p(k)$ is computed by solving the linear system given by

$$
B(k) p(k)=-g(k)
$$

where $g(k)$ is the gradient vector, and $B(k)$ represents one of a sequence of matrices which form an approximation to the true Hessian matrix previously described. When dealing with objective functions that are inherently imprecise and expensive to evaluate, obtaining the true Hessian matrix may be difficult or prohibitive because of the cost. The approximate Hessian matrix $B(k)$ may be thought of as the Hessian of a quadratic model of the objective function. With the search direction $p(k)$ computed, the next step in the process, known as the linear (or one-dimensional) search, is performed. This is essentially an attempt to step to the minimum of the quadratic model of the objective function, based on the accuracy of the current gradient and curvature information. This is denoted by setting [where $\alpha(k)$ is the linear search step size] 


$$
x_{i}(k+1)=x_{i}(k)+\alpha(k) p(k), \quad i=1,2, \ldots . . N
$$

and

$$
g_{i}(k+1)=g_{i}\left[x_{i}(k+1)\right], \quad i=1,2, \ldots, N
$$

Here $x_{1}(k+1)$ is the new approximate solution vector of the problem. Now, the gradient estimations are evaluated again in the new region of the design space to which the solution has progressed. Again, either forward- or central-difference formulas may be used depending on the perceived accuracy of the gradients, and on whether a sufficient decrease in function value has been obtained during the last linear search procedure.

The final step in this simplified quasi-Newton process is an update to the approximate Hessian matrix $B$ to reflect changes (and presumably increased information) about the nature of the objective function. This update procedure is quite complex, and must satisfy certain conditions to maintain the positive definiteness and symmetry of the approximate Hessian, even in the presence of round-off error. The condition of positive definiteness is necessary to insure that each new search direction $p(k)$ will in fact be a descent direction. Schematically, this update procedure is given by

$$
B(k+1)=B(k)+U(k)
$$

where $U$ is the update matrix. This update matrix is designed such that the new approximate Hessian $B(k+1)$ satisfies the important quasi-Newton condition, that is,

$$
[g(k+1)-g(k)]=B(k+1)[x(k+1)-x(k)]
$$

On the first optimization iteration of a new problem, $B(0)$ can be set to the identity matrix until the first update can be formulated. 


\section{Termination Criteria}

For the special case of a quadratic objective in $\mathrm{N}$ dimensions, the quasi-Newton algorithm should in theory converge to the minimum in $\mathrm{N}$ iterations. However, when dealing with an imprecise objective function that displays somewhat discontinuous behavior, the marginal accuracy of the calculated gradients will generally preclude such rapid convergence. Still, a great deal of progress may have been made toward significantly decreasing the function value in these $N$ iterations, as well as reducing its sensitivity to further changes in the solution vector (small gradient norm). Thus, user supervision may be necessary to determine the most valid termination criteria based on the current solution status.

\section{Finite-Difference Step-Size Selection}

The proper selection of appropriate finite-difference step sizes is vital to the success of the optimization algorithm. All of the information about the nature of the objective is derived from the computed gradients, and the accuracy of the gradients is largely dependent on the finite-difference interval size. For problems such as transonic flow design in which limited function precision is available, the selection of the proper step size will permit the most effective use of the available precision. These step sizes will no doubt vary slightly from one problem to another and, therefore, should be recomputed for each new design case. 
In the new design program developed here, problem-adaptive finite-difference step sizes are computed automatically by routine FDSTEP (finite-difference step size) at the outset. This routine is based on algorithm FD in Reference 26, and was implemented in the work by Kenne11y [20]. In essence, FDSTEP begins by evaluating the objective over certain intervals whose lengths are determined based on the user's estimate of the function precision and the scaling of the problem. By making estimates of the cancellation and round-off errors present in the trial gradients computed, FDSTEP selects a final step size that should be near optimal for the problem. This process is repeated for each of the design variables; usually two to four function evaluations are required per variable. Thus, two- to four $-\mathrm{N}$ function evaluations are necessary before optimization can begin. Although this may appear costly, the vastly increased performance afforded by using these optimal step sizes is well worth the cost of computing them. Additionally, as a by-product, FDSTEP also provides accurate gradients for use by QNMDIF in the first optimization iteration, as well as estimates of the diagonal elements of the approximate Hessian matrix $B(0)$. Thus, a great deal of vital information about the objective function is accumulated by FDSTEP before optimization is begun. This should aid QNMDIF in getting off to a good start, and relieve the user of the difficult task of manual step-size estimation. Indeed, it is felt that the success of the present design work is due in large part to the optimality of the step sizes so computed. 
The details concerning the operation of FDSTEP are beyond the scope of this report and will not be presented. Instead, the interested reader is referred to the cited references. 
CHAPTER IV

\section{AERODYNAMIC DESIGN PROGRAM DEVELOPMENT}

The major part of this research effort was in the coupling of the transonic flow-analysis program with the numerical optimization algorithm previously described. This coupling is not straightforward and requires a careful integration of the two relatively unrelated components of the design tool. Fortunately, both TWING and QNMDIF are well structured and designed to be adaptable to new applications. The new application of this work will be the first time that a transonic wing flow-field solution program of the speed of TWING has been integrated with a quasi-Newton method optimization algorithm to create a fully three-dimensional design tool.

The essential communication between the flow solver and the optimization program is established through the objective function to be minimized, and the $\mathrm{N}$-dimensional vector of design variables. The definition of these two vital entities is generally the major factor in the performance obtained from the design program. It is here that the present work will explore two new approaches: the objective function will be derived directly from the aerodynamic force coefficients computed by the analysis program, and the design variables will control the wing-surface geometry by the use of a new spline-fitting technique. This is in contrast to previous 
methods in which the objective function was based on the difference between the computed pressure field and one that was specified as a target. This reverts the operation of an NOD program to a pseudoinverse mode - more flexibility is present than with a true inverse method. This mode of operation, referred to as the $\mathrm{C}_{\mathrm{p}}$-objective mode, is also possible with the present design program, and an example using this option is presented. Also, the design variables in the previous methods served merely as magnification coefficients of the terms in a series of shape functions. Here, a more direct technique for geometry perturbation will be described.

The essential function of the main driving routine of the design program is operation sequencing and design management. There are several steps that must be completed during each design cycle, and within the context of a computer program this amounts to calling the appropriate subroutines at the proper time. An outline of the steps in a typical design iteration is illustrated as follows:

1. Wing geometry generation (G80)

2. Grid generation about the current geometry (GRGEN3)

3. Transonic-flow solution (TWING)

4. Evaluation of objective function

5. Compute new geometrical perturbation (QNMDIF)

6. Check convergence and solution; return to Step 1 The names in parentheses refer to the computer programs that handle the particular steps. $\mathrm{G} 80$ is a wing-alone geometry-generation 
program based on a wing-body geometry generator developed by Sobieczky [24]. GRGEN3 is the grid-generation subroutine within TWING. Each of these programs is modified somewhat from its original form in order to utilize better its capabilities in an iterative design mode. A brief description of the most important steps in the design iteration will be given in the following sections.

\section{Wing Geometry Generation}

Although the GRGEN3 routine within TWING can establish general wing geometries itself, it was felt that use of a separate special-purpose geometry generator would add additional versatility in the design program. A special feature of the $G 80$ routine is the manner in which the airfoil sections that make up the wing are defined. From relatively few (or alternatively, many) coordinate points, a cubic spline interpolation is used to create a wel1defined airfoil. This is done on an expanded scale, which reduces the curvature sufficiently so that oscillations in the spline curve may be avoided. This particularly aids in defining a smooth leading edge, even though when rescaled down to the correct size the radius may still be quite small. This spline-support point method lends itself naturally to the new geometry perturbation technique used in the design program. This will be discussed in a later paragraph. The geometry generation program G80 accepts three input airfoils used as defining stations for the wing. The location of the stations is fixed at the root and tip, with the third main or break station located at some intermediate point specified by the user. 
The spline-support points defining these three input airfoils are then used to spline-fit many more points to aid in well defining the section shapes. The wing planform size and shape must also be specified by the user. This is done indirectly by the use of analytical relations describing generalized curves over a unit interval. The starting and ending points of the curve are specified, as well as its slope, and then an analytical connection fit and blending are used to define the desired shape. Although it seems complex, the technique is actually easy to use and offers a great deal of generality and flexibility. In addition to defining the planform shape, arbitrary twist and dihedral distributions may be specified in the same way.

From the three basic defining airfoils, up to 20 output stations may be requested. Across the span, the section distribution may be varied, as well as the relative influence of each of the three defining stations. The latter is effected through four influence coefficients. The output stations and planform characteristics are then arranged and formatted for use by the grid-generation routine (GRGEN3). The proper specification of such general shape and influence parameters cannot easily be determined without some experimentation, but they are not critical; if in doubt, the user can use standard values initially. Because of this great flexibility, it is possible to optimize the wing planform, as well as its surface shape, in future work. This area of design is largely unexplored. 
The next two steps in the design cycle, namely, grid generation and transonic flow-field solution, have already been discussed in Chapter II. These two steps effectively amount to a function evaluation based on the current solution. The result must next be interpreted by the driving program and converted into an objective function value suitable for use by the optimizer. The question of this interpretation will be treated next.

\section{Evaluation of Objective Function}

As has been stated, the objective function must relate improvements in the aerodynamic quality of the design to the optimizer by a decrease in function value. The aerodynamic drag computed by the flow solver is an obvious choice; however, its use has been limited because of the anticipated low reliability of its calculation, especially when computed by an inviscid potential method. In theory, the drag calculated by this type of flow solver should be composed of two parts: induced drag, or the drag owing to lift, and shock-wave drag. The shock-wave drag in inviscid flow manifests itself as a momentum deficit essentially, and this effect is enhanced physically because of boundary-layer separation, if the shock is of more than moderate strength. Thus, for efficient operation in the transonic regime, it is the component of wave drag that must be minimized (the induced drag is generally a function of lift and spanwise lift distribution only, and is determined by planform).

A more sophisticated approach than simple drag minimization might be maximization of the so-called transonic efficiency 
parameter. This is expressed as the product of the free-stream Mach number and the lift-to-drag ratio. This quantity is directly related to the cruise range of a turbojet-powered aircraft operating in the transonic regime. Also, this objective will prevent the optimizer from reducing the drag simply by reducing lift or thickness. Here, it will be the optimizer's task to increase the liftto-drag ratio of the wing design through more complex means. Evidently, the best that may be hoped for is a design in which a wing shape is found that is capable of supporting shock-free flow at the specified Mach number, while preserving as much of the original lift as possible. Since a specified flight Mach number is usually given, it will be removed from the objective function and held constant. Thus, it will be the optimizer's task to maximize the lift-to-drag ratio of the configuration (or, operationally, to minimize its reciprocal).

What has been described is effectively the same objective as that of shock-free design methods. However, there is one important difference: shock-free methods will fail to produce any answer if no shock-free solution can be found. In general, for a given freestream Mach number and wing-loading condition (lift coefficient), there may in fact be no shock-free flow solution. Nevertheless, it may be possible to redesign the surface shape such that the shockwave strength is appreciably weakened, while the lift is reduced only slightly (or even increased). Also, these changes may be shown to require minimal changes in wing-section thickness and surface 
shape. Thus, no severe penalties in structural design or fuelcarrying capacity need be incurred. For these reasons, the dragto-lift ratio minimization of an initial wing configuration at a fixed Mach number will be defined to be precisely the objective of this work.

Mention must be made, however, of another objective function that may be utilized: the so-called $\mathrm{C}_{\mathrm{p}}$-objective function. It is simply a measure of the difference between the pressure field given by the current design and a target pressure distribution that is specified. The use of this objective will drive the optimizer toward shaping the wing surface such that the computed pressure field matches that of the target. Operated in this manner, a numerical optimization design program becomes an expensive substitute for an inverse design method. Of course, a great deal more flexibility and user intervention is available. One result showing the implementation of this objective is presented. The remainder of results will deal strictly with the unexplored technique of drag-to-lift ratio minimization.

\section{Geometrical Perturbation Technique}

The technique used in the present work is designed to reduce the number of design variables required to explore a sufficiently broad design space, and is directly related to physical changes in wing-section shape. The computer time required for a given design is directly proportional to the number of design variables that must 
be used. Thus, the minimum number of variables that will allow the design objective to be met should be used. In a design case study on the Lockheed C-141B aircraft, the shape-function-series technique used in Reference 14 used a total of 120 design variables. In the present study, the new spline-support point movement (SSPM) technique yielded an excellent design at similar conditions using only 12 geometrical design variables.

The SSPM technique is so named because the three defining airfoil sections used in the wing geometry program are derived from a spline-fit of several spline-support points. Over the region where surface reshaping is desired, a few support points can be located, and control of their vertical position is given to the optimizer. Thus, subregions of an entire wing may be easily redesigned by modifying only a certain part of the defining airfoil. Over the remainder of the alrfoil section, many fixed points are used to control accurately the spline. Thus, a smooth new airfoil shape will be defined for each new location of the movable support points. This procedure will be illustrated in Chapter V.

It has been mentioned that although constraints may be imposed on the solution with certain types of optimization algorithms, it is generally preferable to formulate the problem such that constraints become unnecessary. The SSPM technique of the present work in some sense imposes implicit constraints on the design by restricting the class of airfoil that may be defined. Because much of the airfoll surface shape is held fixed by the 
immovable points, the portion where reshaping is permitted must still conform in a reasonable way to the rest of the surface. Thus, large deformations and discontinuous curvature should be shunned by the optimizer. Any unacceptable perturbations to the flow field will manifest themselves as increases in drag and, hence, increases in the objective function. Hence, the need for the imposition of formal constraints should be reduced with the SSPM method. Having described some of the theory of aerodynamic configuration design using numerical optimization procedures, the remainder of this report will present four actual wing design cases. The general objectives of each case will be discussed, as well as some details of the procedure involved. The choice of spline-support points will also be explained. 


\section{CHAPTER V}

\section{WING DESIGN CASE RESULTS}

The new transonic wing-configuration design program combining TWING with the numerical optimization routine QNMDIF has been described and will now be applied to practical wing-design problems. The design program as a unit will now be referred to as TWING/QNM. Three interesting wing geometries have been selected among aircraft in operation today: the Lockheed $C-141 B$ military transport, and the Gates Learjet Model 55 and Cessna Citation III business jets. These three wing configurations all differ appreciably in sweep, aspect ratio, and planform, and also utilize different types of airfoil sections in their definition. The flight conditions at which new improved designs are sought are chosen to be typical of the actual operating conditions for these aircraft. For the Cessna Citation wing, a design at a slightly higher Mach number and lift coefficient than normal was sought. The achievement of the specified objectives of each design case should prove the integrity of the new design program TWING/QNM. Some details of the procedure, as well as an explanation of the design objectives and interpretation of the results will now be given for each of the three wing designs studied. 
Lockheed C-141B Wing Design

The Lockheed C-141B military transport is a four-engine aircraft with a high-mounted wing that was designed primarily for the transport of cargo. The simplified isolated wing geometry that will be treated in this design is shown in Figure 2. Geometrical specifications of this wing include a leading-edge sweep of $27.7^{\circ}$ and an aspect ratio of 7.89 . A linear twist distribution was imposed on the geometry such that the incidence angle varies from $+1.0^{\circ}$ at the root to $-0.5^{\circ}$ at the tip. No dihedral was specified. Having defined the planform characteristics, the next step in formulating the design problem is the selection of the three initial or baseline airfoil sections to be used as the defining stations. Here, all three sections were selected to be the same GA(W)-2MOD airfoils. This airfoil is based on the low-speed $G A(W)-2$ airfoil used on general aviation aircraft, but with a slightly modified upper surface for better high-speed performance. It was selected for its excellent low-speed, high $C_{I_{\max }}$ characteristics [27]. It is a fairly thick airfoil at $13 \%$, with moderate aft cambering. The modified $G A(W)-2 M O D$ airfoil used in this work has been thinned to be $11.8 \%$ thick.

The next step in setting up a design run is choosing the location of the fixed and movable spline-support points. Because improvements in the supercritical performance of the wing are the primary objectives, only modifications to the upper surface of the airfoil will be permitted. Further, the region of shape modification will be restricted to essentially that region wetted by 

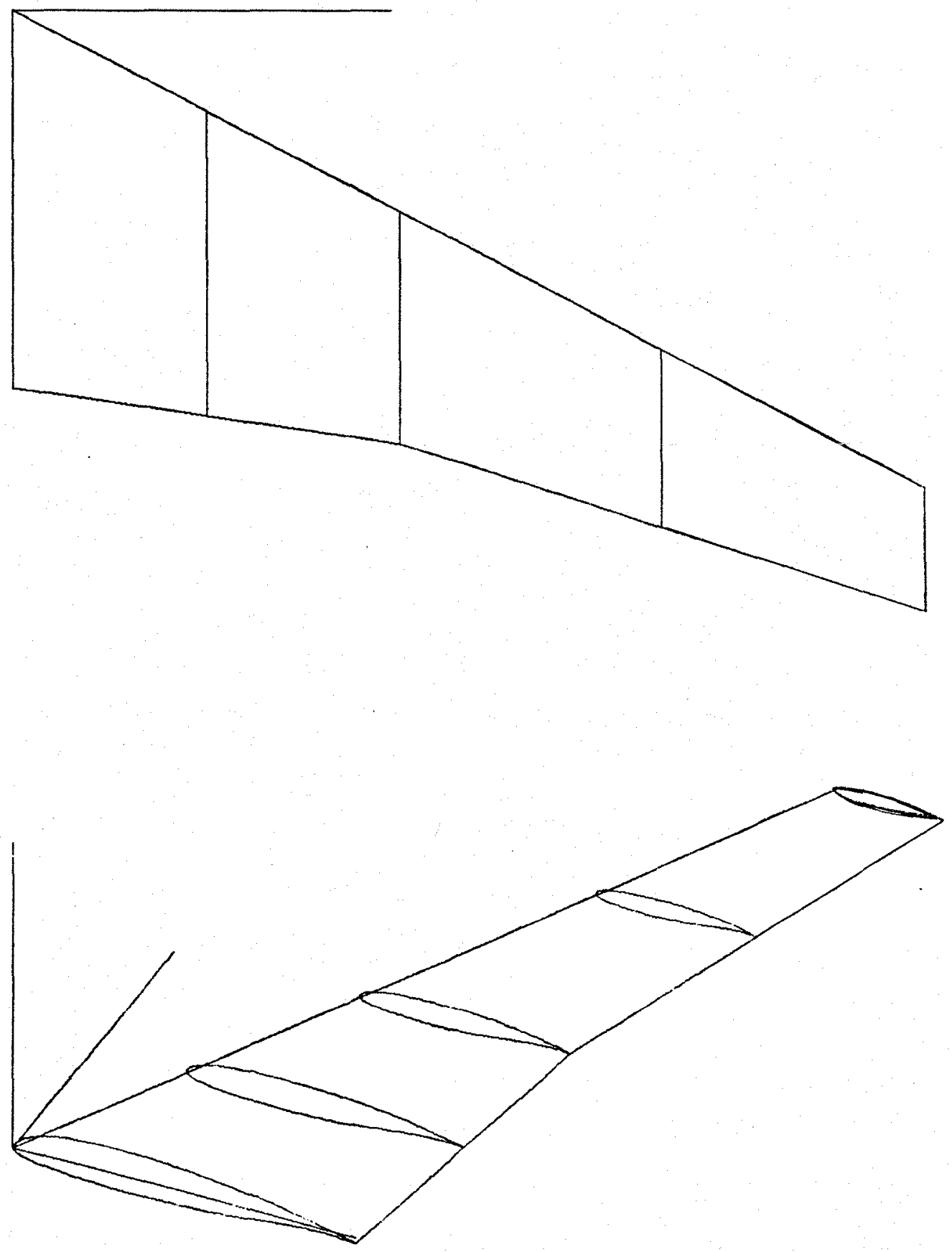

Figure 2. Two views of the Lockheed $\mathrm{C}-141 \mathrm{~B}$ wing geometry with aspect ratio 7.89 and leading-edge sweep of $27.7^{\circ}$. (a) Planform view; (b) isometric view. 
supersonic flow, as it has been shown in a two-dimensional shockfree airfoil design problem by Cosentino [9] that small changes in shape here may have a great effect on the entire flow. Therefore, four spline-support points are positioned somewhat arbitrarily over the region of the upper surface where supersonic flow may be expected. These four points are designated to be movable, and the remainder of the airfoil is defined by a number of fixed points spaced much closer together. This arrangement is illustrated in Figure 3. Note that the important leading-edge shape and radius, as well as the trailing-edge angle, are preserved by the clustering of fixed points. With this geometrical parameterization, modifications permitting improved transonic performance are facilitated, yet reasonable constraints on the class of airfoil that may be defined have been imposed because of the location and number of fixed coordinate points.

Having specified completely the starting wing configuration and the method of geometrical perturbation, the final step before the actual numerical design can begin is the selection of the flight conditions, that is, the free-stream Mach number and wing lift coefficient. For this first design attempt, a flight Mach number of 0.77 and a lift coefficient for the initial configuration of 0.60 were chosen. These conditions are typical, yet reasonably moderate. Because the design objective function to be minimized is the dragto-lift ratio (for the purposes of scaling, this value is then multiplied by 100 to bring it to order unity), it may be expected that changes in both the lift and drag coefficients will appear as 


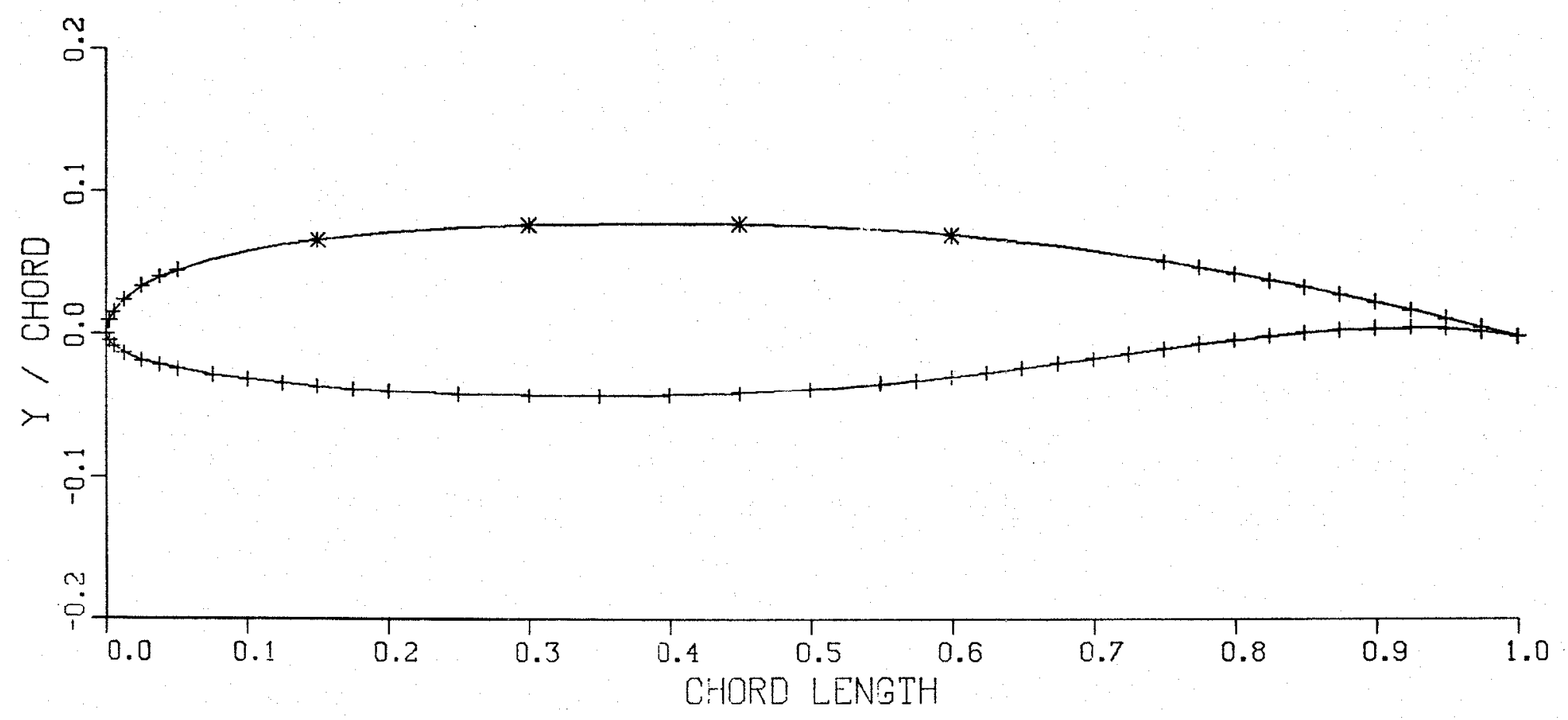

Figure 3. Location of the fixed $(+)$ and movable (*) spline-support points on the GA(W)-2MOD airfoil: 
the design progresses. Thus, the starting lift-coefficient value will not in general be the same as that achieved on the optimized design. This situation could perhaps be rectified by altering the definition of the objective function. This will not be treated further here.

A complete design problem has now been specified, and the actual design can begin. Note that there are four movable points on each of the three defining wing stations, a total of 12 design (or decision) variables. As the design progresses, the vertical positions of these spline-support points are shown, along with the value of the objective function. Because the use of the computed drag coefficient is relatively unexplored, the design termination criteria should not be determined from the objective function and gradients alone. User intervention is required. Inspection of the wing-surface shapes and resulting pressure distribution is imperative, and should be done periodically throughout the design process. The improvement in lift-to-drag ratio, combined with smooth pressure distributions with weakened shocks and physically reasonable airfoil shapes are together sufficient evidence to declare a design successful.

In the Lockheed $\mathrm{C}-141 \mathrm{~B}$ wing design case, termination was initiated after 12 optimization iterations were completed. Although the optimized airfoil shapes and pressures were reasonable, some waviness in the pressure distribution was noted, It was found that the new surface did not match the rest of the original airfoil shape because of the location of the fixed points. To correct this, the 
amount of airfoil surface fixed by the imnovable points was reduced, and the airfoil shape based on the optimized location of the movable points and fewer fixed points was redefined with a new spline fit. This process amounts to nothing more than a smoothing of the new shape defined by the optimization process. The C-141B planform with these final optimized and smoothed airfoils was then analyzed in a separate IWING solution. The results of this solution, superimposed with the original airfoils and pressure distributions, are shown at various span stations in Figures 4 through 8 . These results indicate that a design attempt using the computed lift-todrag ratio as the objective function has been quite successful. An almost shock-free solution has been found, corresponding to essentially a minimization of the wave drag component in the total computed drag. This has been accomplished using only 12 geometrical variables, and required just $1.43 \mathrm{hr}$ of Cray X-MP CPU time. The fully three-dinensional nature of the problem is evidently handled adequately by modifying only three wing stations independently (the two stations between the root, break, and tip stations are interpolated). A summary of the design is presented in Table 1 .

Table 1. Summary of Lockheed C-141B Wing Design

\begin{tabular}{lccc}
\hline & Original wing & New wing & Percent change \\
\hline$C_{L}$ & 0.585 & 0.558 & -4.62 \\
$C_{D}$ & 0.00967 & 0.00723 & -25.23 \\
$L / D$ & 60.535 & 77.264 & +27.64 \\
\hline
\end{tabular}




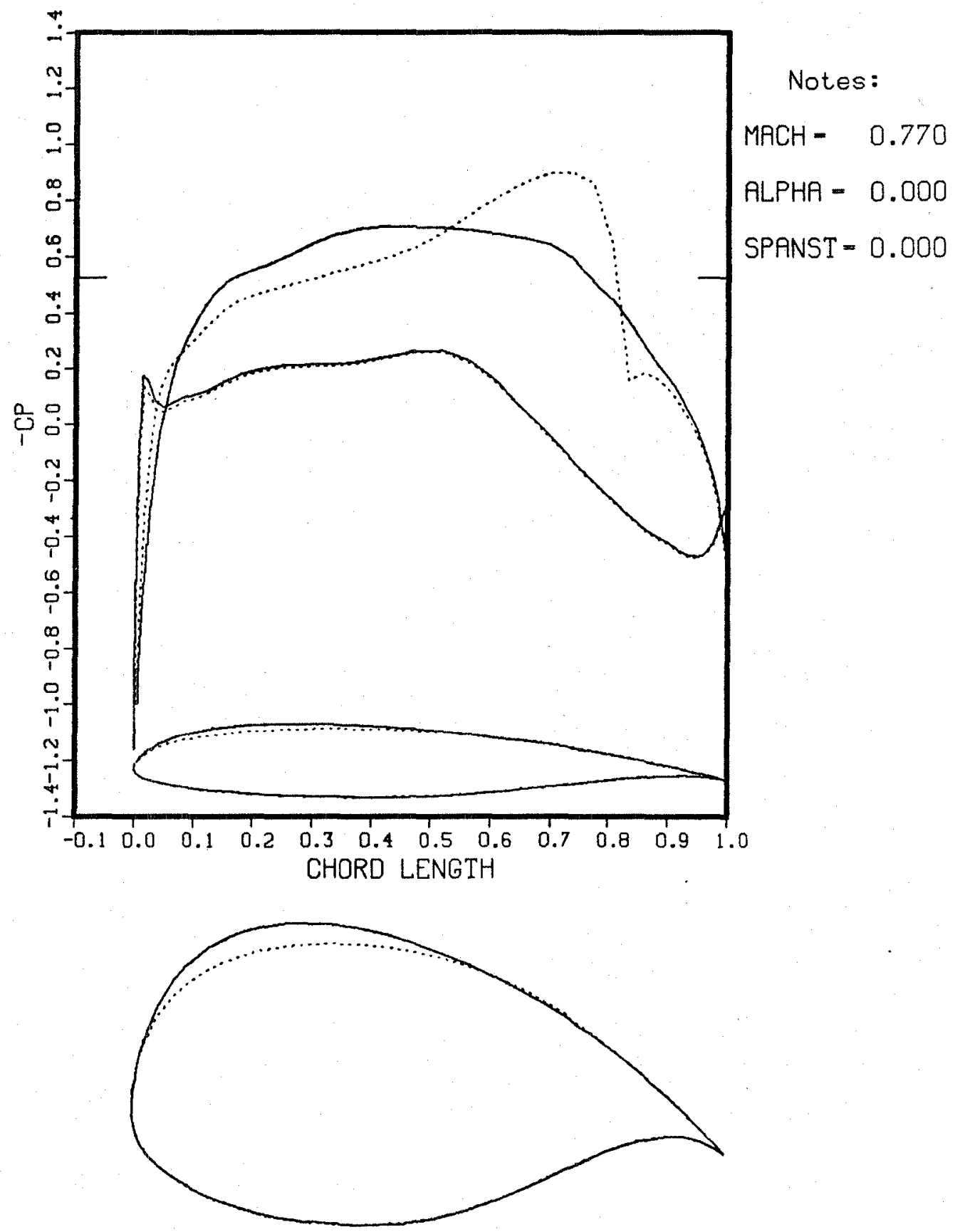

- AIRFOIL SHAPE AND PRESSURES AFTER OPTIMIZATION RUN ...... ORIGINAL GA(W)-2MOD AIRFOIL SECTION AND PRESSURES

Figure 4. TWING/QNM drag-to-lift ratio minimization result, Lockheed C-141B wing design: root span station. 


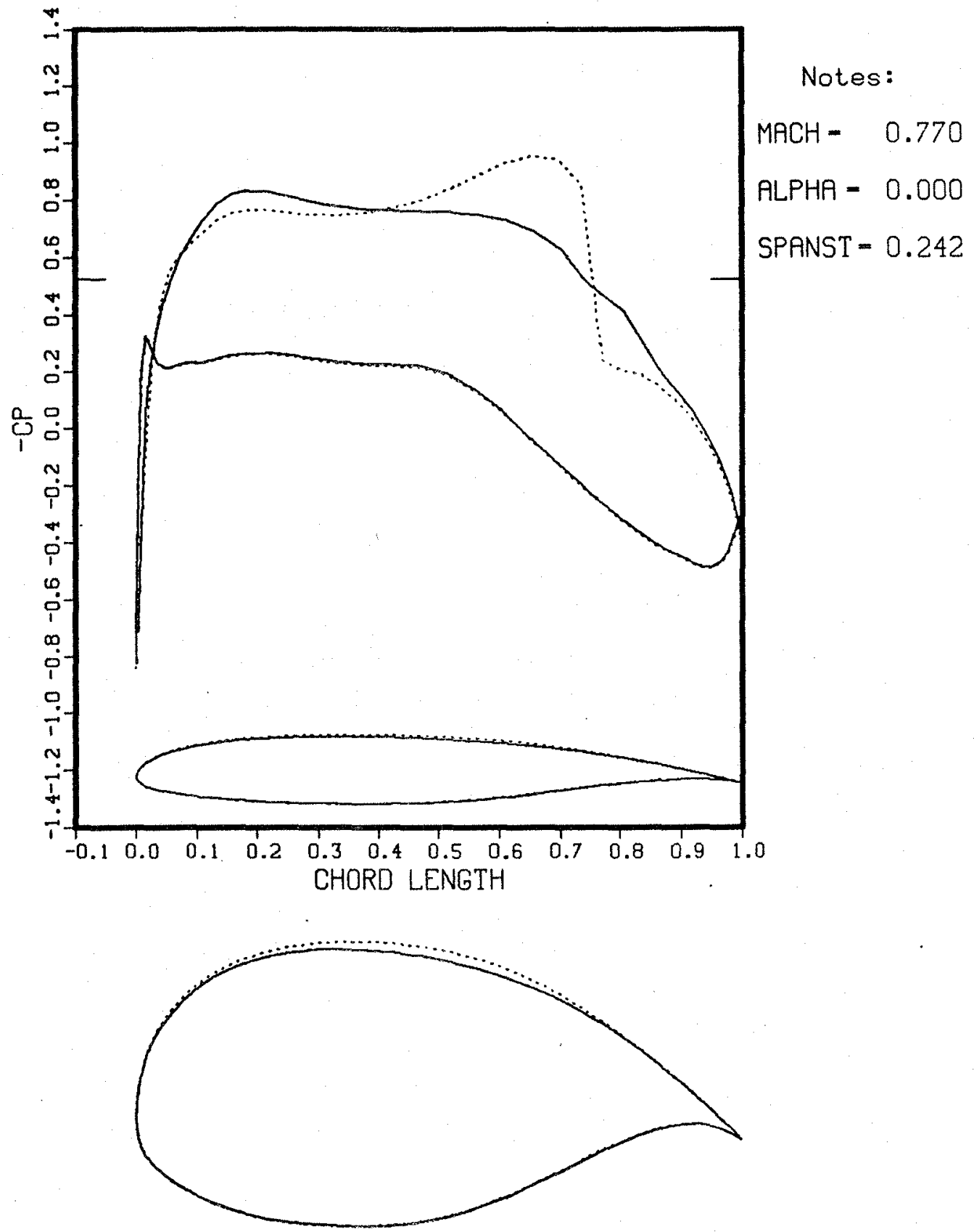

- AIRFOIL SHAPE AND PRESSURES AFTER OPTIMIZATION RUN

....... ORIGINAL GA(W)-2MOD AIRFOIL SECTION AND PRESSURES

Figure 5. TWING/QNM drag-to-lift ratio minimization result, Lockheed C-14IB wing design: $24.2 \%$ span station. 

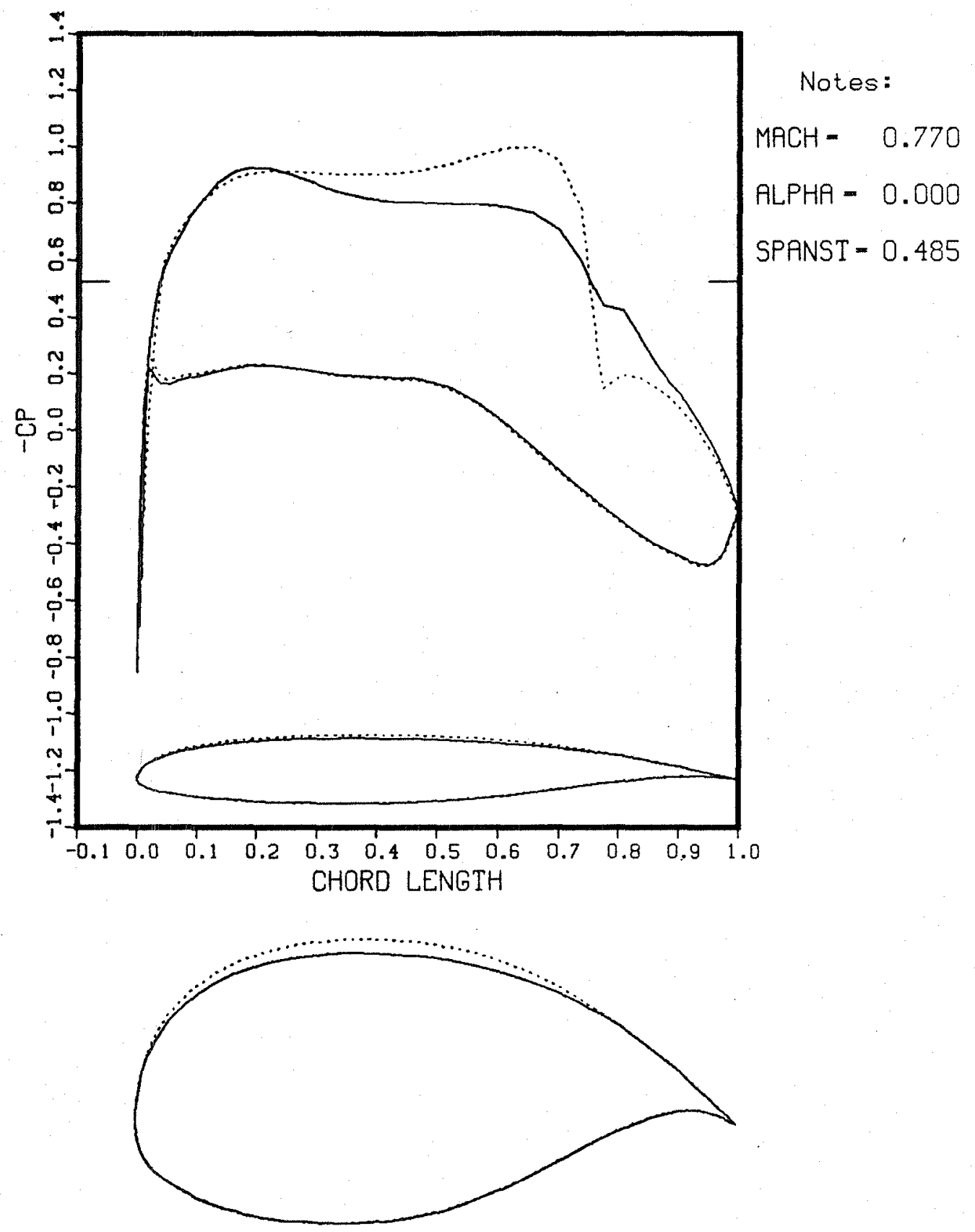

— AIRFOIL SHAPE AND PRESSURES AFTER OPTIMIZATION RUN ...... ORIGINAL GA(W)-2MOD AIRFOIL SECTION AND PRESSURES

Figure 6. TWING/QNM drag-to-lift ratio minimization result, Lockheed C-141B wing design: $48.5 \%$ span station. 

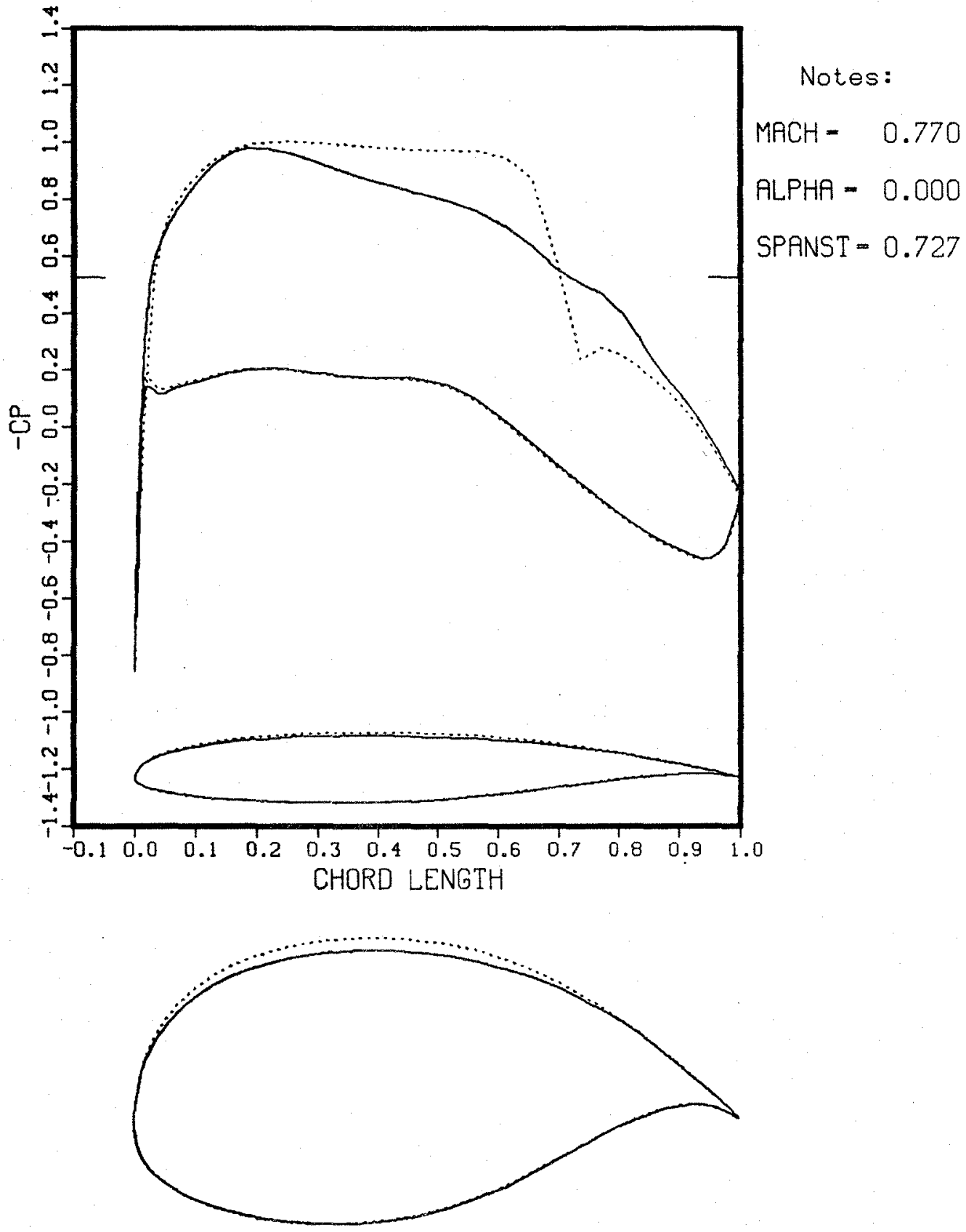

- AIRFOIL SHAPE AND PRESSURES AFTER OPTIMIZATION RUN

...... ORIGINAL GA(W)-2MOD AIRFOIL SECTION AND PRESSURES

Figure 7. TWING/QNM drag-to-lift ratio minimization result, Lockheed $\mathrm{C}-141 \mathrm{~B}$ wing design: $72.7 \%$ span station. 

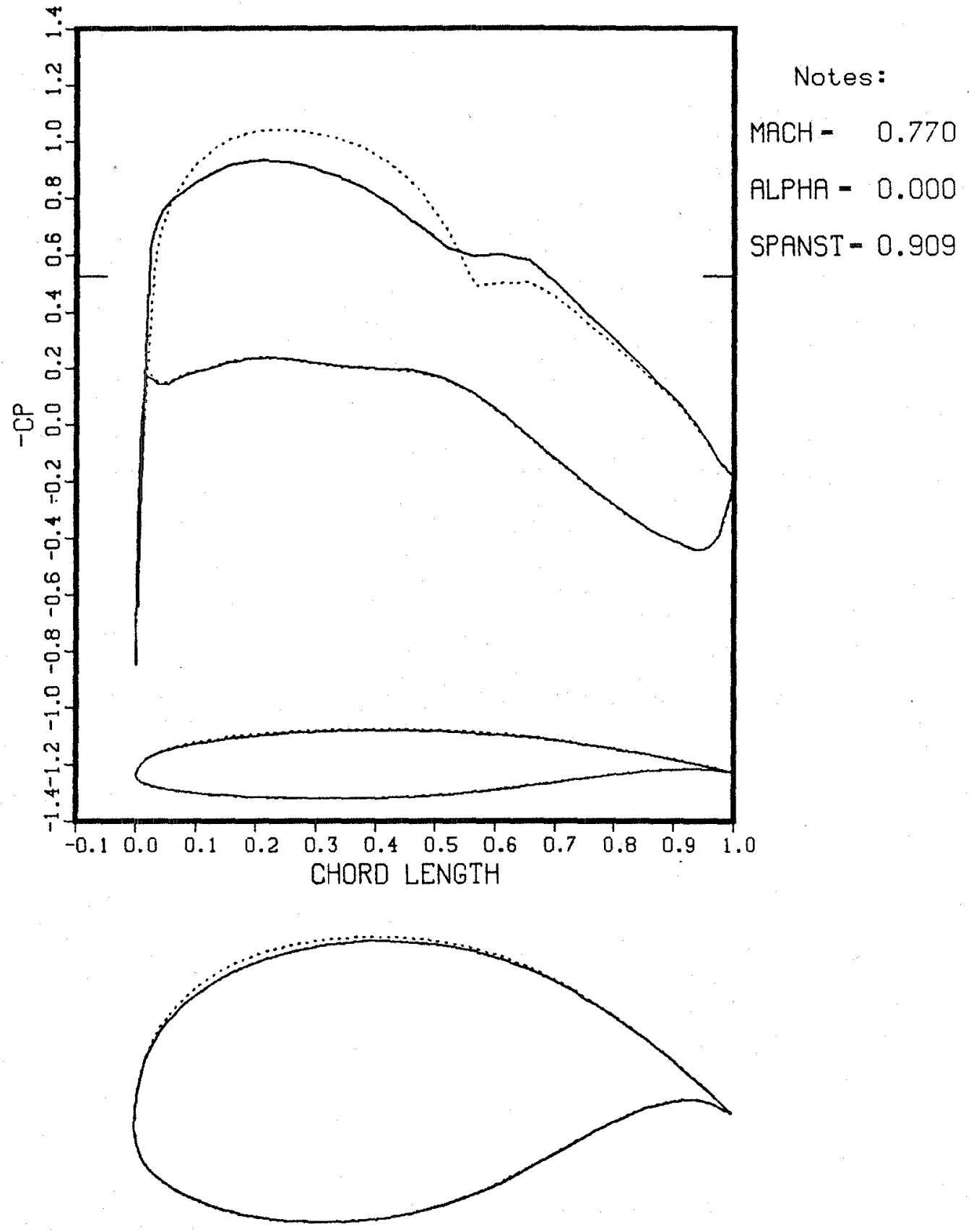

- AIRFOIL SHAPE AND PRESSURES AFTER OPTIMIZATION RUN ORIGINAL GA(W)-2MOD AIRFOIL SECTION AND PRESSURES

Figure 8. TWING/QNM drag-to-lift ratio minimization result, Lockheed C-141B wing design: $90.9 \%$ span station. 
Figure 9 displays the convergence characteristics of this design by plotting the objective function (the drag-to-1ift ratio multiplied by 100 for scaling) versus the number of flow analysis solutions (function evaluations) performed. The relatively wild behavior over the first third of the curve is actually the correct functioning of subroutine FDSTEP in estimating appropriate finitedifference step sizes for each of the 12 variables, and evaluating preliminary gradients and the diagonal elements of the approximate Hessian matrix. Thus, the first 37 function evaluations are performed by FDSTEP in order to "learn" about the nature of the function and the shape of the design space. The accumulated information is then passed to QNMDIF, and the first linear search step is initiated. As can be seen in Figure 9, this first step results in an immediate decrease in the objective. QNMDIF then initiates a recomputation of the gradient vector, and calculates a new updated search direction. The next linear search results in another decrease, and the process is repeated. As the optimization design progresses, the amount of improvement (or decrease of objective) at each line search step is reduced, and eventually the function levels to some constant value. This may be interpreted physically as an elimination (as best as possible) of the wave- drag component, leaving a constant level of induced drag.

Figure 10 reveals the overall drag characteristics of both the original and optimized wings. The coefficient of drag (scaled by a factor of 100) is plotted at several Mach numbers for each wing (the lift coefficient is held fixed at approximately 0.56). As can 


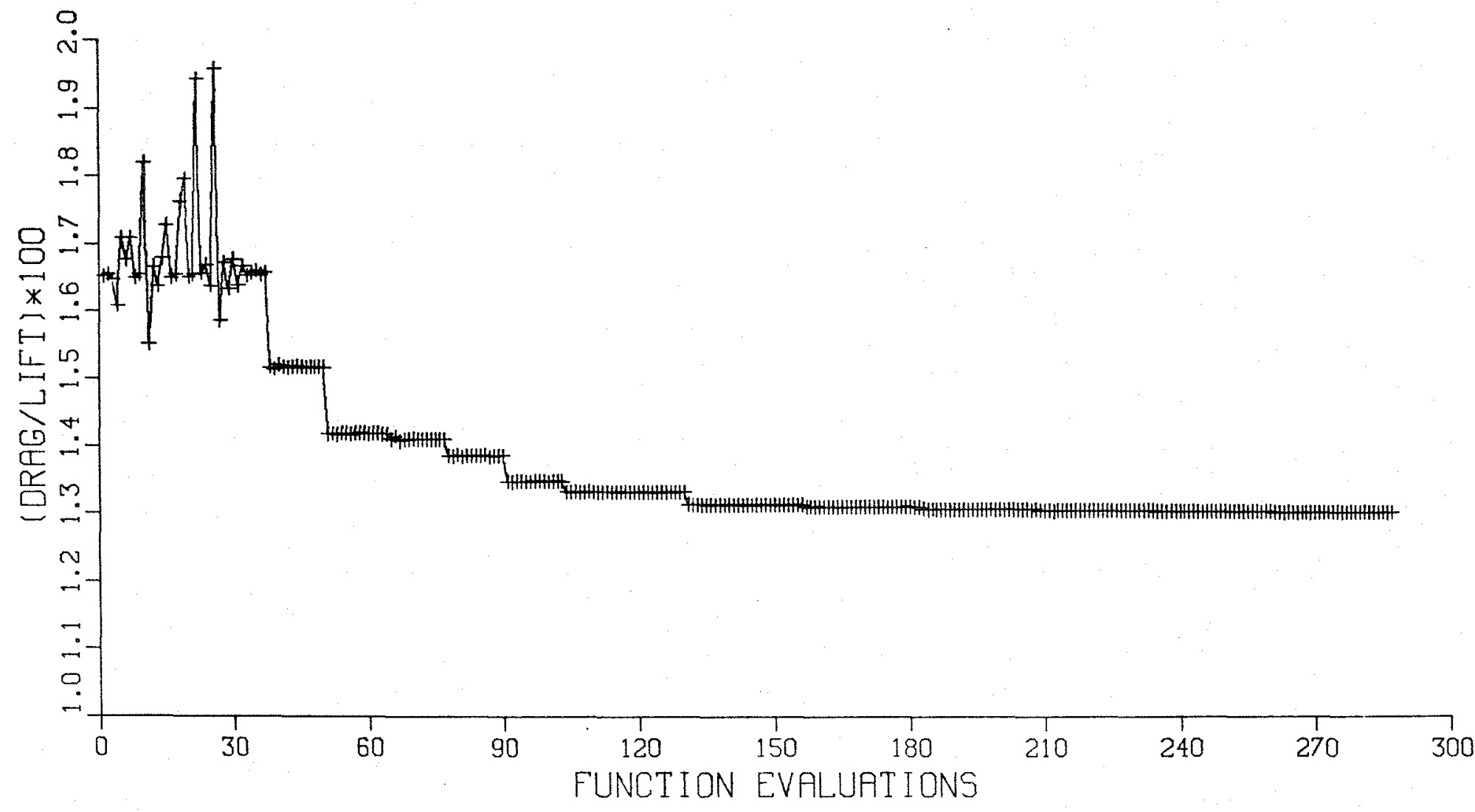

Figure 9. Objective function $\left(C_{D} / C_{1} \times 100\right)$ versus the number of TWING flow solutions: C-141B wing. 


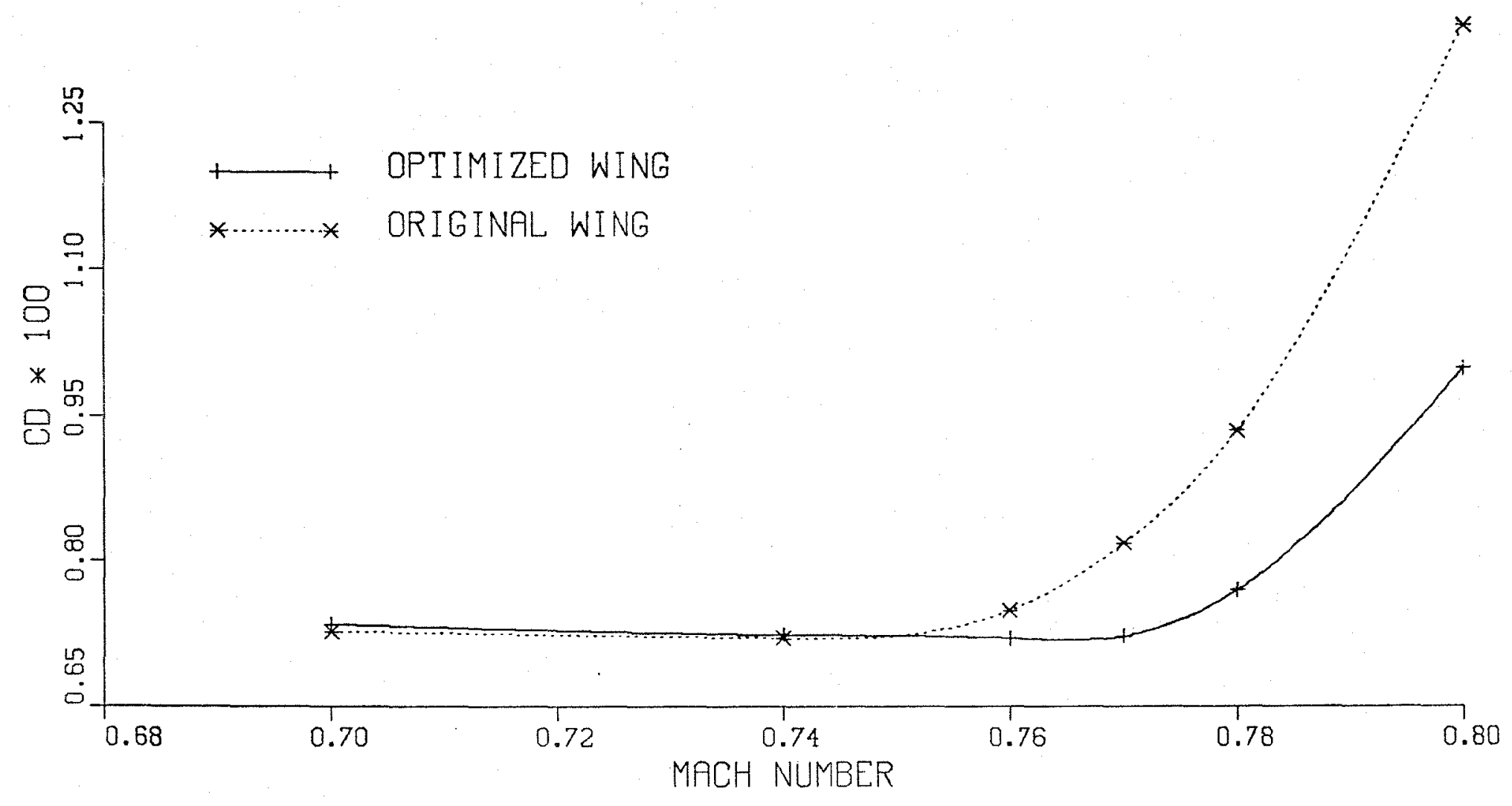

Figure 10. Coefficient of drag $(\times 100)$ versus Mach number for the original and optimized wing. 
be seen, the optimized wing displays superior drag-rise characteristics as the Mach number is increased, yet does not suffer any undesirable off-design behavior at the lower Mach numbers. One of the objectives of this design, namely, an improvement in the transonic efficiency of the wing (or an increase in its lift-to-drag ratio), has been adequately satisfied. This has been accomplished by using only 12 design variables, tight flow solver convergence, and just $1.43 \mathrm{hr}$ of computer time. This represents a significant advancement in the state of the art of transonic configuration design using numerical optimization procedures.

\section{Gates Learjet Century III Wing Design}

The next wing configuration studied is the Gates Learjet Century III wing as shown in Figure 11. This is the same wing as that used on the Learjet Model 55, with the winglets removed. It has a fairly low leading-edge sweep of $15.9^{\circ}$, and an aspect ratio of

6.72. A cubic twist variation has been specified such that the angle of incidence varies from $0^{\circ}$ at the root to $-1.0^{\circ}$ of washout at the tip; the dihedral angle is $2.5^{\circ}$. The NACA $64_{1} \mathrm{~A} 212$ airfoil was used to define all three input stations. This airfoil was selected because it is similar to several Learjet airfoils currently in use, and is not significantly aft-loaded. Aft loading may create undesirable control-surface moments, and this is particularly a problem on aircraft with purely mechanical actuators.

For this design, only three movable spline-support points were selected. These are shown in Figure 12. Choosing just three 

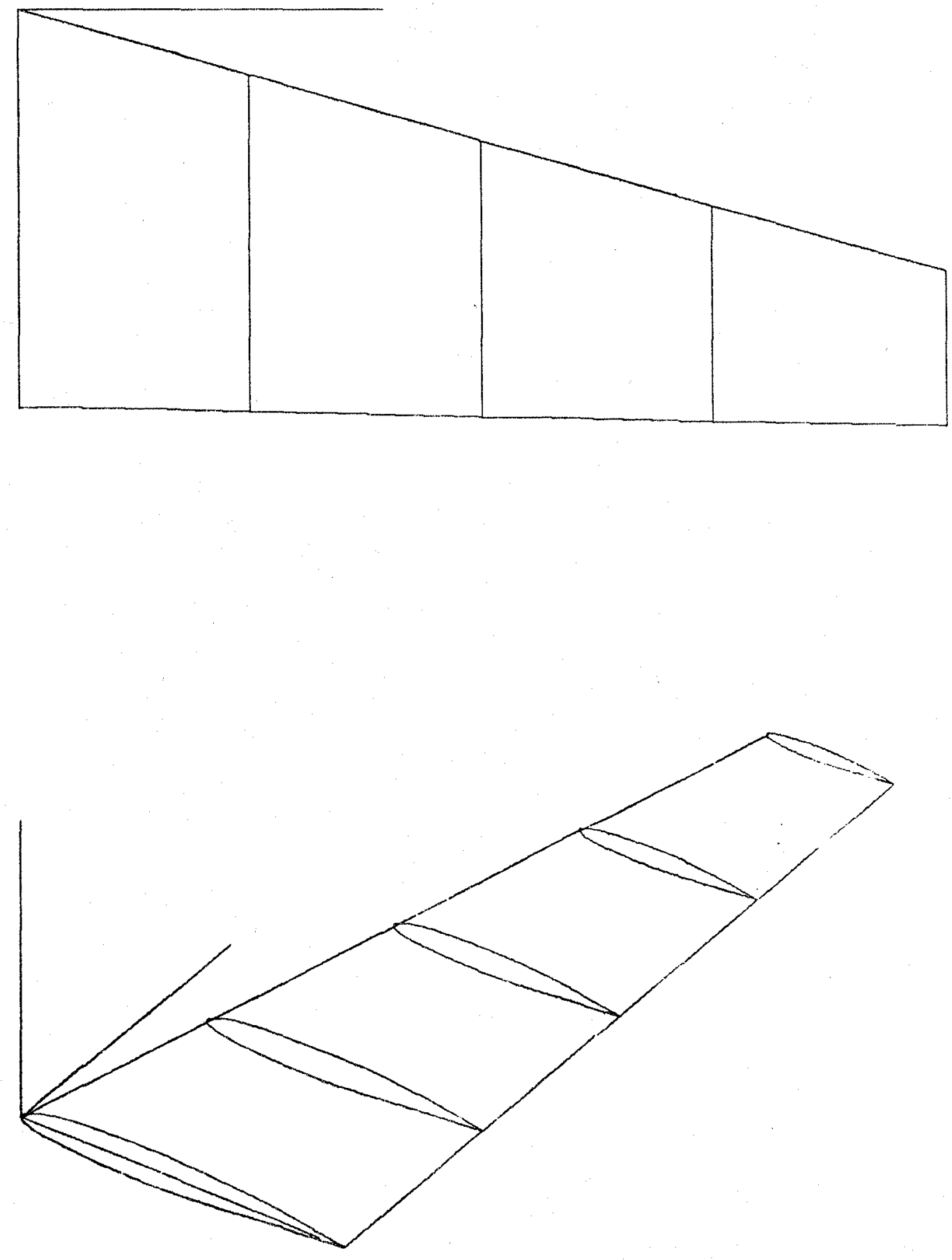

Figure 11. Two views of the Gates Learjet Century III wing geometry with aspect ratio 6.72 and leading-edge sweep of $15.9^{\circ}$. (a) Planform view; (b) iscmetric view. 


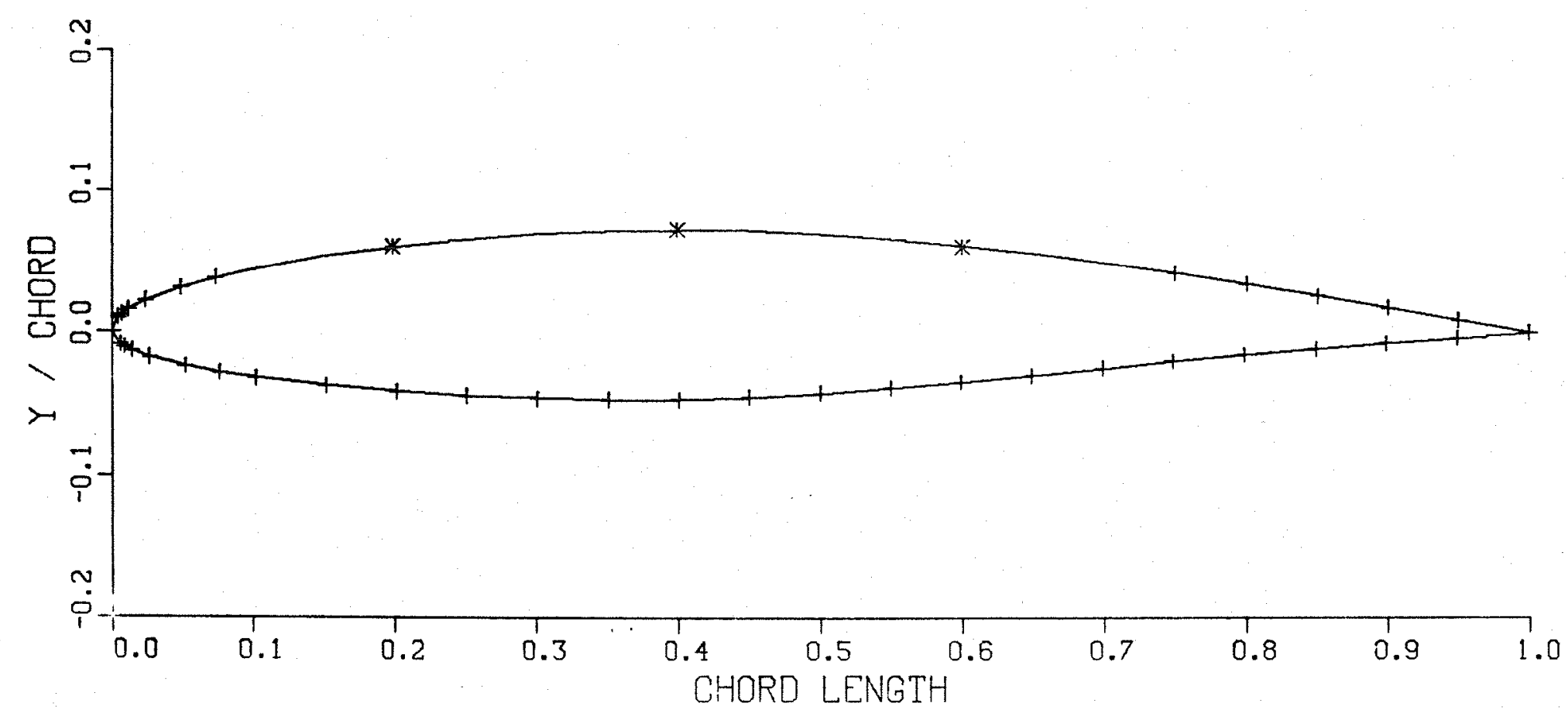

Figure 12. Location of the fixed $(+)$ and movable $(*)$ spline-support points on the NACA $64_{1}$ A212 airfoil. 
points at each station will reduce the total number of design variables from the 12 in the previous case to 9 . This should increase the computational efficiency of the design, and demonstrate whether a sufficiently broad design space is possible with only three movable points on each airfoil. The points were again located over approximately the region wetted by supersonic flow. A free-stream Mach number of 0.78 and a lift coefficient of 0.48 were chosen as the initial conditions to formulate a practical design problem. The progress of the design was inspected after the third, sixth, and ninth optimization iterations by plotting the new airfoil shapes and pressure distributions across the span. After the ninth design iteration, the process was terminated. The resulting wing design was then analyzed with a separate TWING computation. The new shapes and pressures are compared with the original NACA $64_{1}$ A212 airfoils and pressures in Figures 13 though 17 . In this design case, although a shock-free solution has not been found, the shock wave strength has been significantly reduced and is now acceptably weak (the root station is shock-free). The section thickness at midspan has been reduced from $11.0 \%$ to approximately $10.3 \%$ as a result of the design reshaping. From the strong shock present on the original airfoil, and the apparent inability of the optimizer to locate a shock-mfree configuration, it may be concluded that the original airfoil is rather inefficient for operations at high transonic speeds. Despite this, a substantial improvement has been made in the lift-to-drag ratio of this wing. The total computer time 

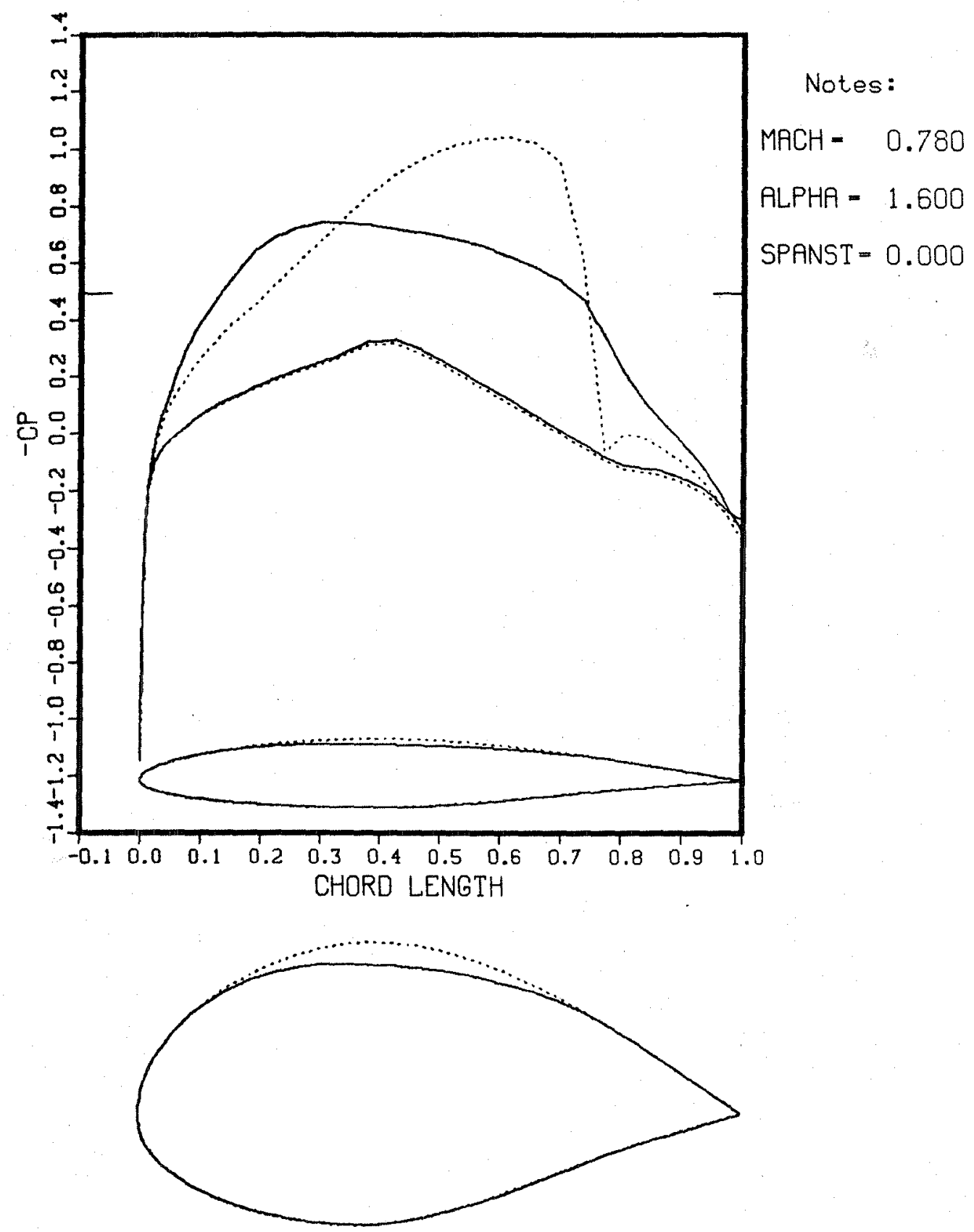

- AIRFOIL SHAPE AND PRESSURES AFTER OPTIMIZATION RUN

...... ORIGINAL NACA 64(1)-A212 AIRFOIL SHAPE AND PRESSLIRES

Figure 13. TWING/QNM drag-to-1ift ratio minimization result, Gates Learjet Century III wing: root span station. 

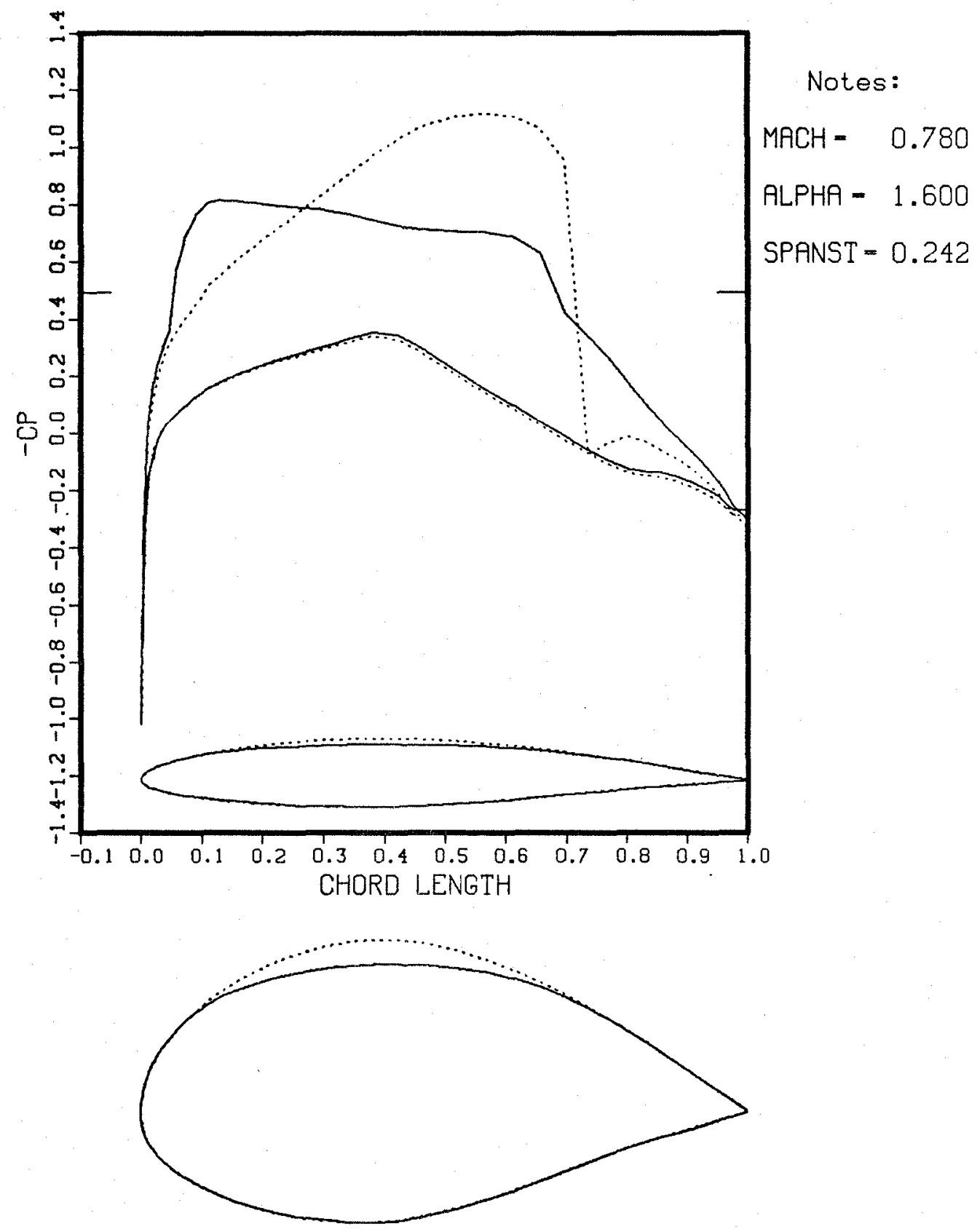

- AIRFOIL SHAPE AND PRESSURES AFTER OPTIMIZATION RUN ORIGINAL NACA 64(1)-A212 AIRFOIL SHAPE AND PRESSURES

Figure 14. TWING/QNM drag-to-lift ratio minimization result, Gates Learjet Century III wing: $24.2 \%$ span station. 

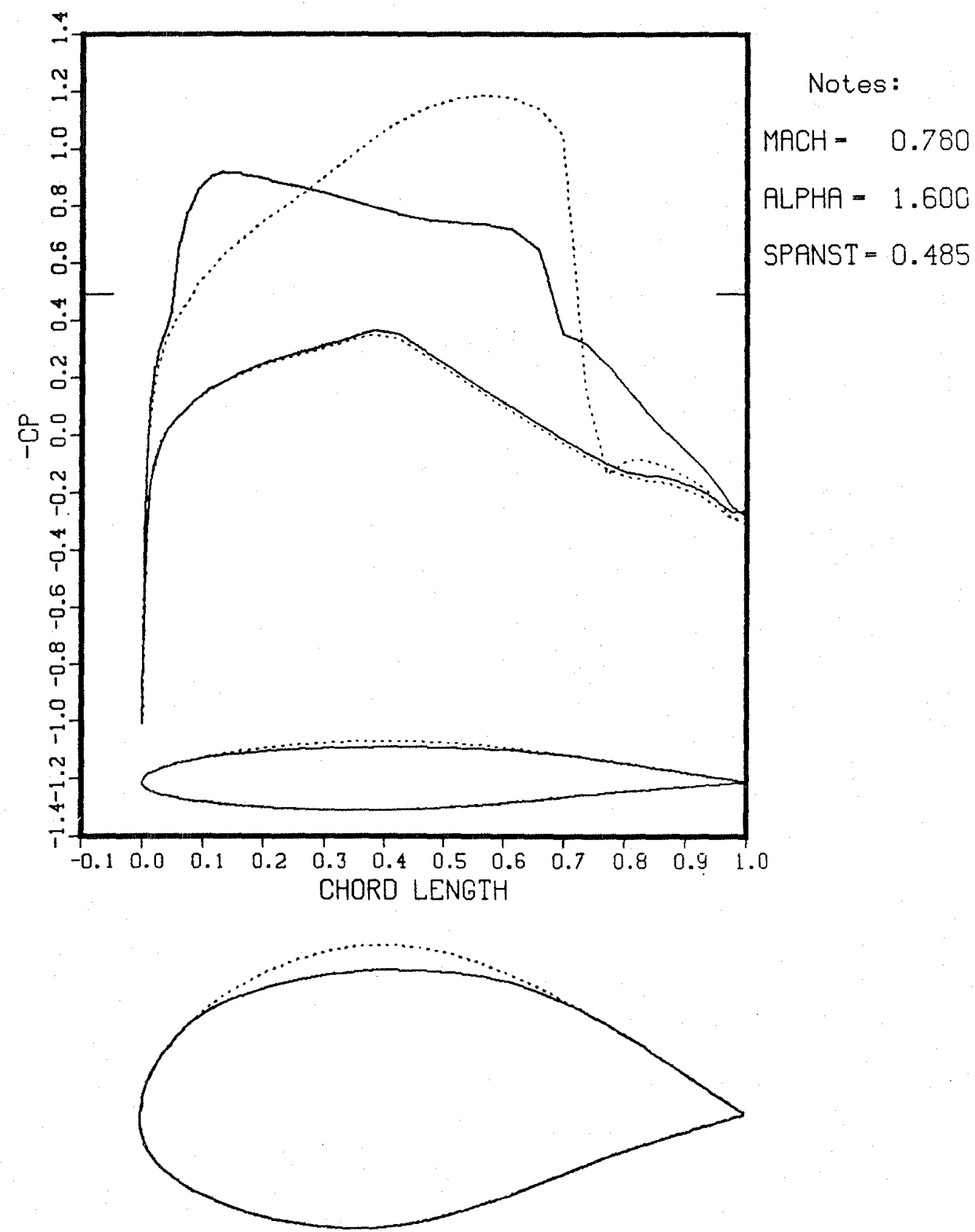

\section{AIRFOIL SHAPE AND PRESSURES AFTER OPTIMIZATION RUN} ORIGINAL NACA 64(1)-A212 AIRFOIL SHAPE AND PRESSURES

Figure 15. TWING/QNM drag-to-lift ratio minimization result, Gates Learjet Century III wing: $48.5 \%$ span station. 

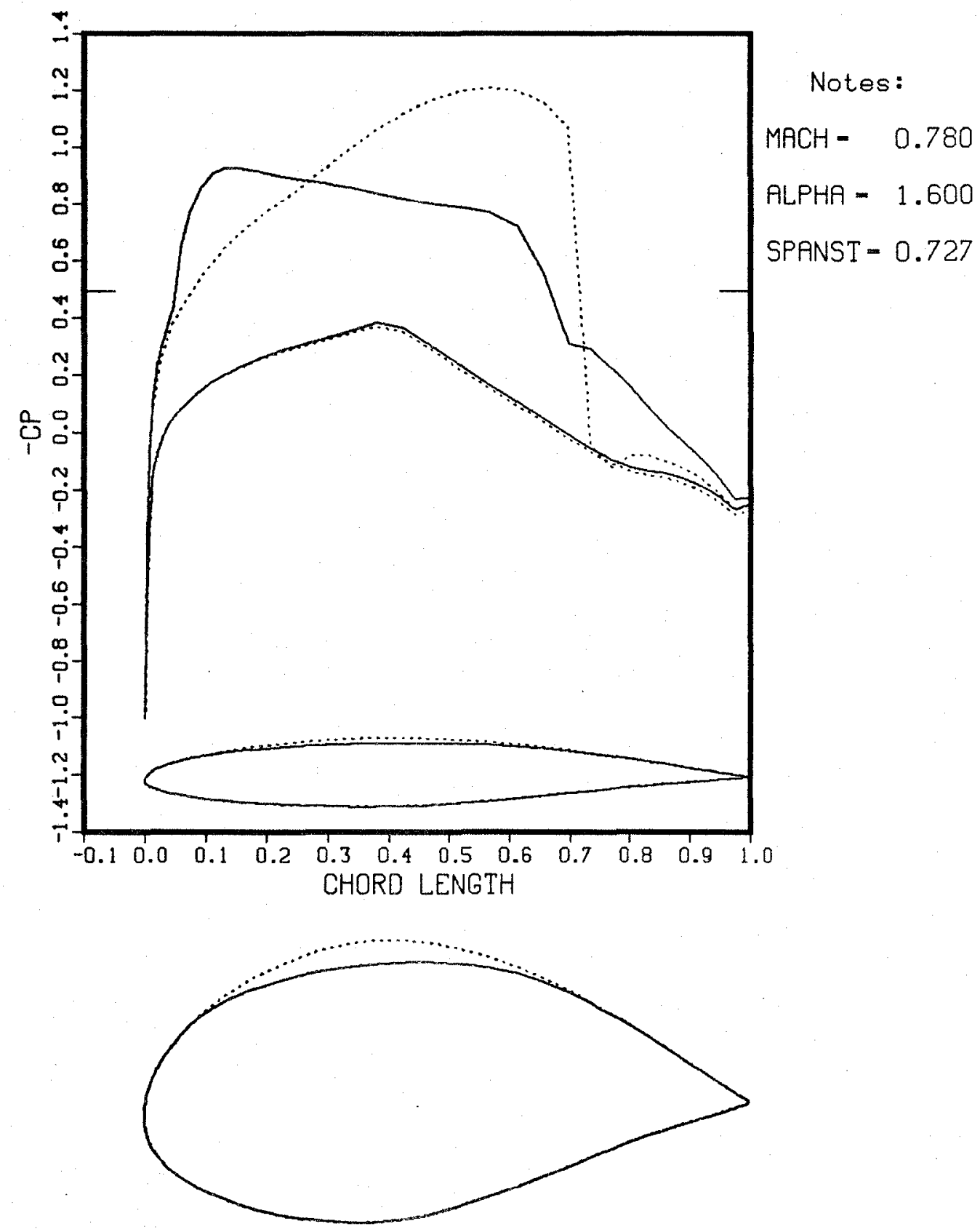

- AIRFOIL SHAPE AND PRESSURES AFTER OPTIMIZATION RUN

...... ORIGINAL NACA 64(1)-A212 AIRFOIL SHAPE AND PRESSURES

Figure 16. TWING/QNM drag-to-lift ratio minimization result, Gates Learjet Century III wing: $72.7 \%$ span station. 

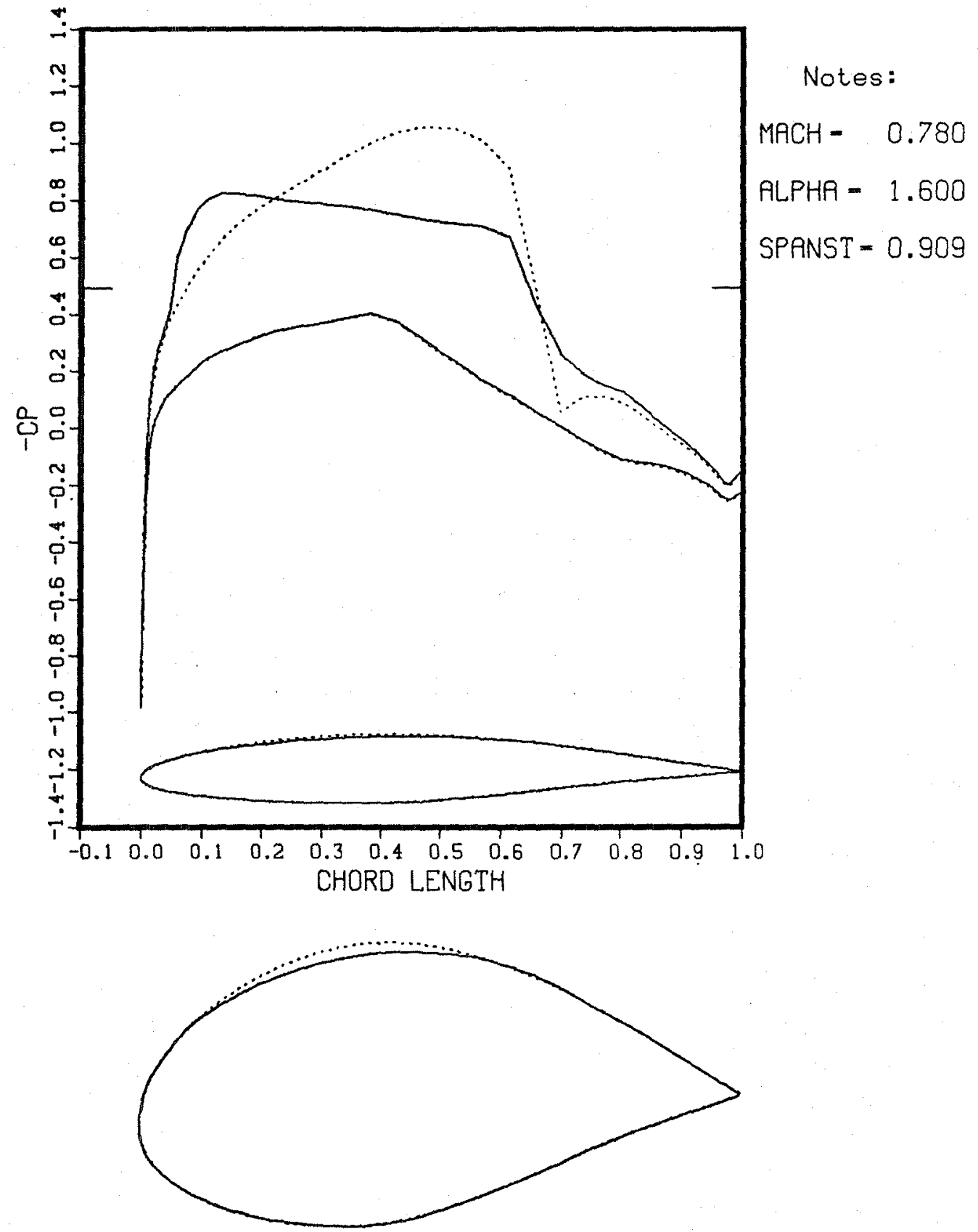

- AIRFOIL SHAPE AND PRESSURES AFTER OPTIMIZATION RUN ...... ORIGINAL NACA 64(1)-A212 RIRFOIL SHAPE AND PRESSLIRES

Figure 17. TWING/QNM drag-to-1ift ratio minimization result, Gates Learjet Century III wing: $90.9 \%$ span station. 
required for this design was $1.05 \mathrm{hr}$. A summary of the results of this design is presented in Table 2.

Table 2. Summary of Gates Learjet Century III Wing Design

\begin{tabular}{cccc}
\hline & Original wing & New wing & Percent change \\
\hline$C_{L}$ & 0.481 & 0.410 & -14.76 \\
$C_{D}$ & 0.01853 & 0.0060 & -67.62 \\
$L / D$ & 25.950 & 68.210 & +162.85 \\
\hline \hline
\end{tabular}

From Table 2, it is observed that at the expense of about $15 \%$ of the 1 ift, a $68 \%$ reduction in the inviscid drag count has been effected, and the overall lift-to-drag ratio has been increased by a rather substantial $163 \%$. This is in accord with the large decrease in shock strength displayed at every span station. Figure 18 shows the convergence characteristics for this optimization case. Here the amount of work done by routine FDSTEP is smaller, since there are only nine design variables. After several successful linear search steps (which lead to significant reductions in the objective), the function begins to level off as it did for the C-141B wing design. Although it may appear that the function evaluations from 72 on are wasted effort, as no decrease in the objective is realized, continuation was permitted to verify that this was the best that could be done with the given level of function precision. Because of time restrictions, a study of the coefficient of drag versus Mach number was not performed for this case; however, one may postulate that it would appear qualitatively the same as that for the previous $C-141 \mathrm{~B}$ case (Fig. 10). 


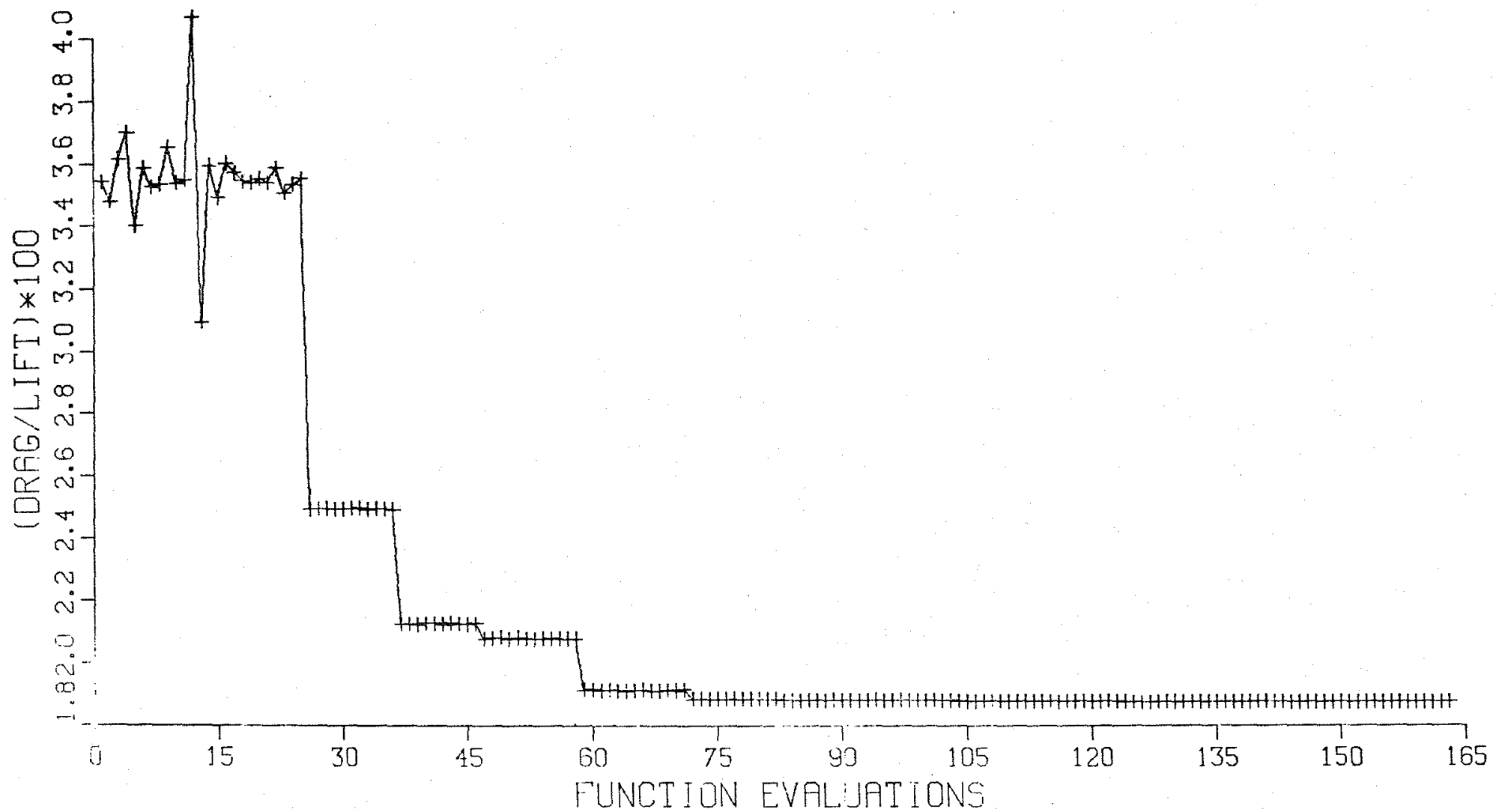

Figure 18. Objective function $\left(C_{D} / C_{L} \times 100\right)$ versus the number of TWING flow solutions: Learjet wing. 
As an aside to the design work presented above, the use of an alternative (and more widely used) objective function will now be explored. The so-called $\mathrm{C}_{\mathrm{p}}$-objective function is defined simply to be a scalar measure of the difference between the computed pressure field and some specified target pressure distribution. The minimum of this function is obviously zero when the computed pressures match the target pressures exactly. A sample design case using the pressure field given by the Learjet planform with GA(W)-2MOD airfoil sections as the target was constructed. A related and yet quite different airfoil was then used as the baseline section for this sample problem. Three movable spline-support points were positioned on the uppex airfoil surface (where the two airfoils differ appreciably), and design commenced. The results of this example are shown in Figures 19 through 21 . The target pressure distribution, the initial pressure difference owing to the new airfoil, and the pressures after optimization design are shown superimposed at three span stations. The baseline wing configuration has been reshaped so as to match the target pressure field closely. Note that no specific design objectives are specified here; this result is included only to serve as an example of the use of this alternative design objective function.

\section{Cessna Model 650 Wing Design}

The final wing design case presented is that of the Cessna Model 650 wing used on the new Citation III aircraft. This geometry is shown in Figure 22. The Model 650 wing has a fairly high 

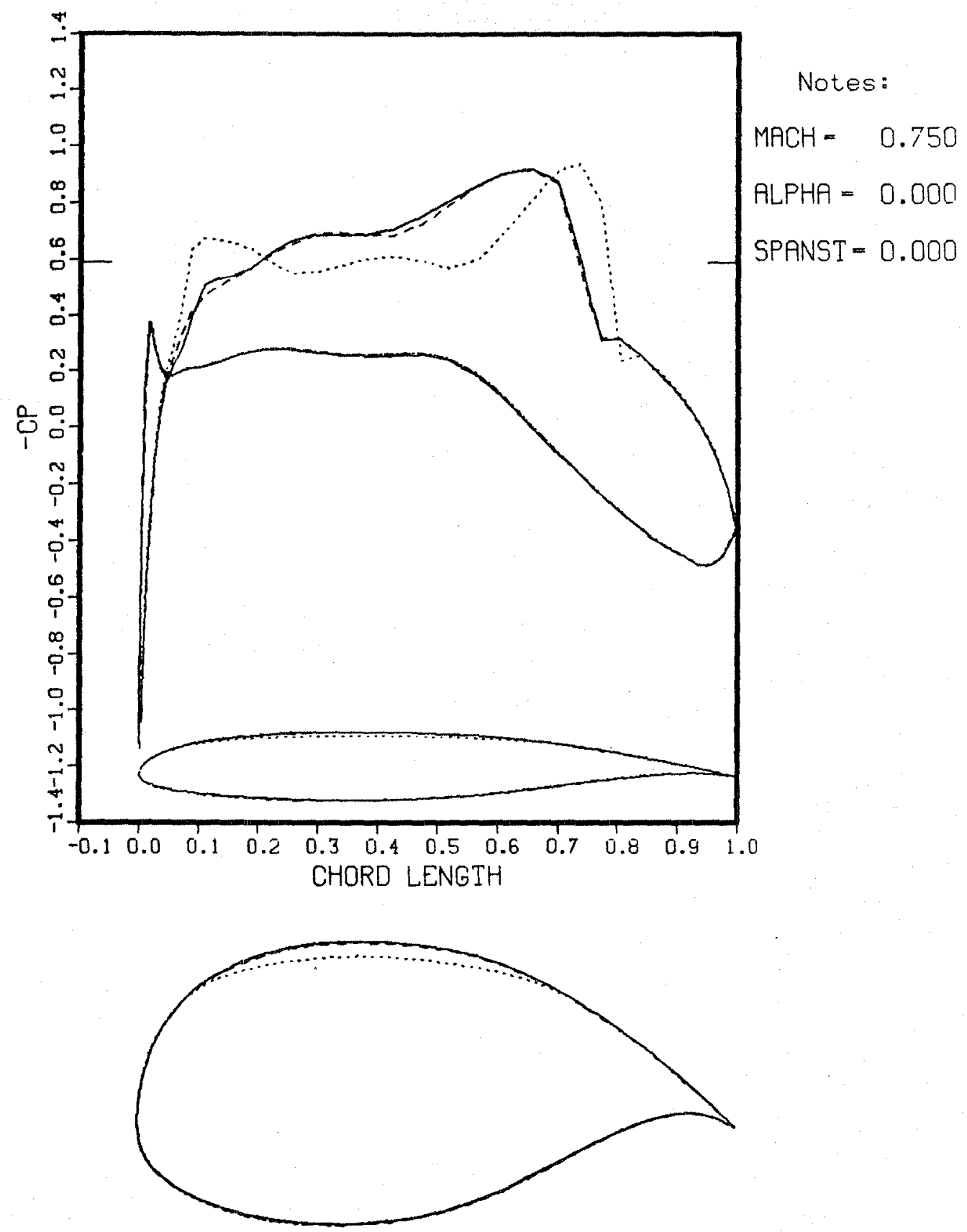

\section{— TARGET PRESSURE DISTRIBUTION GIVEN TO TWING/ONM}

....... INITIRL PRESSURE DIFFERENCE DUE TO AIRFOIL CHANGE

-..- FINAL ACHIEVED PRESSURES AFTER OPTIMIZATION RUN

Figure 19. TWING/QNM pressure-design mode result, Gates Learjet Century III wing planform: root span station. 

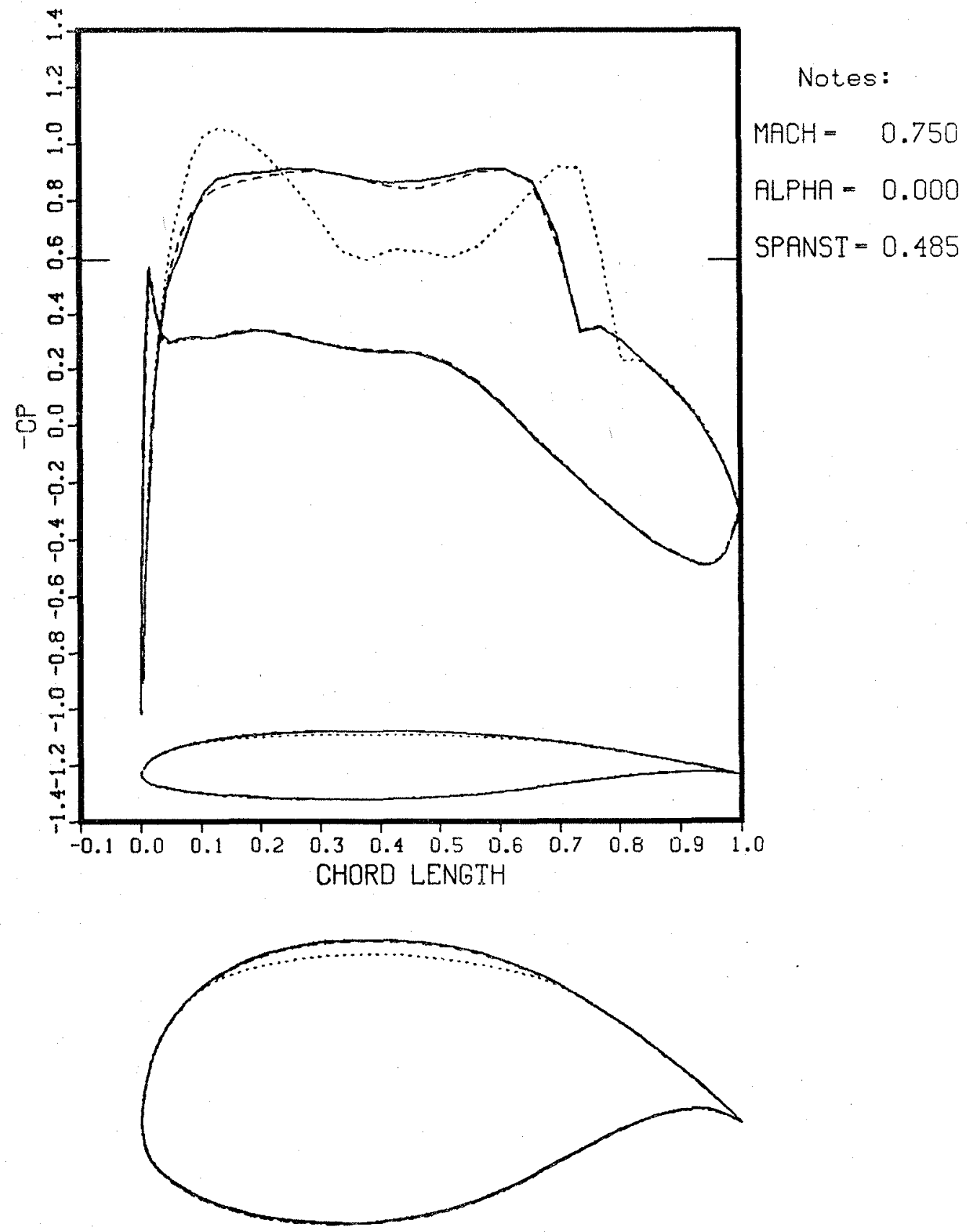

- TARGET PRESSURE DISTRIBUTION GIVEN TO TWING/ONM INITIAL PRESSURE DIFFERENCE DUE TO AIRFOIL CHANGE

$\ldots \ldots$ FINAL ACHIEVEO PRESSURES AFTER OPTIMIZATION RUN

Figure 20. TWING/QNM pressure-design mode result, Gates Learjet Century III wing planform: $48.5 \%$ span station. 

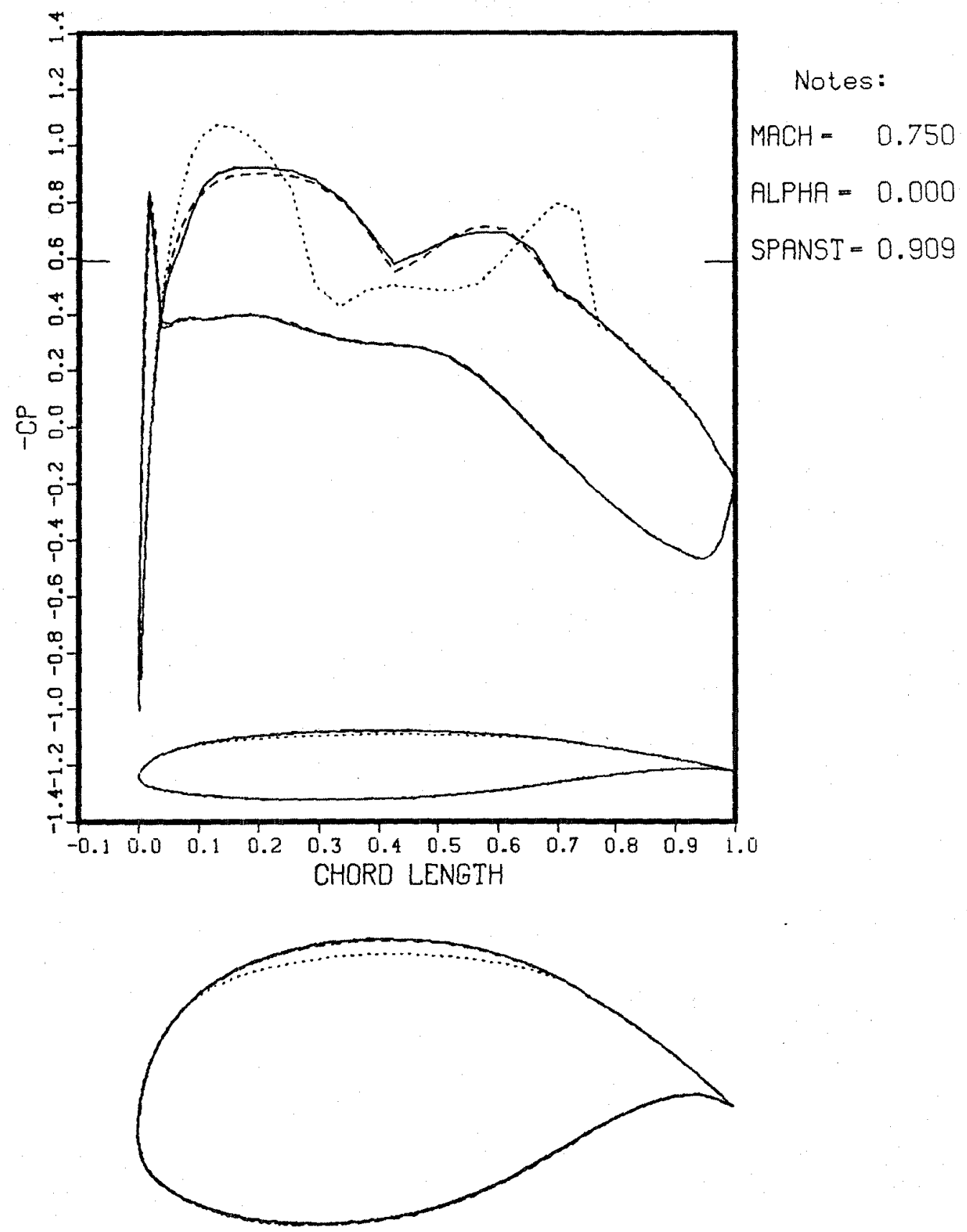

\section{- TARGET PRESSURE DISTRIBUTION GIVEN TO TWING/ONM}

....... INITIAL PRESSURE DIFFERENCE DUE TO AIRFOIL CHANGE

-.- FINAL ACHIEVED PRESSURES AFTER OPTIMIZATION RUN

Figure 21. TWING/QNM pressure-design mode result, Gates Learjet Century III wing planform: $90.9 \%$ span station. 

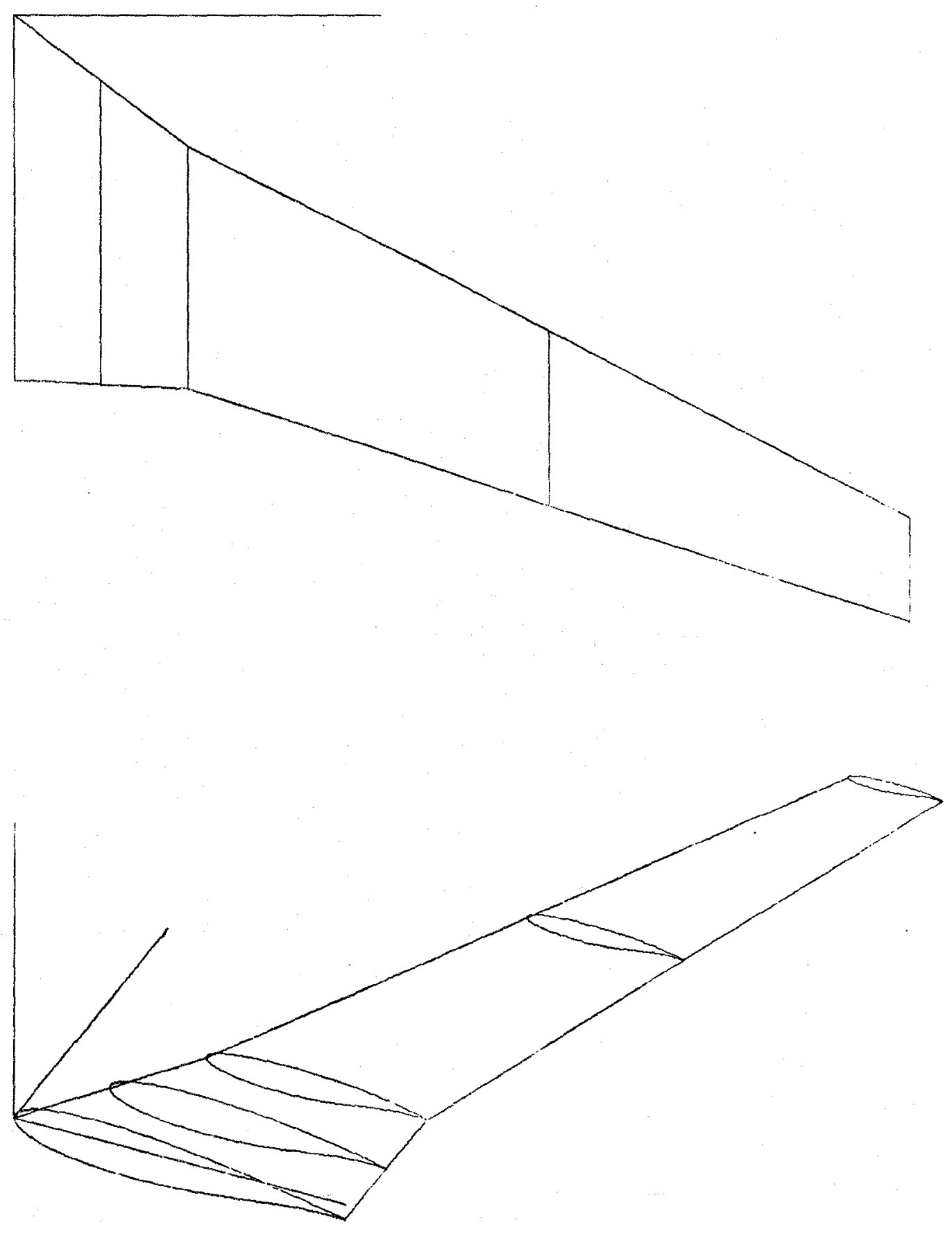

Figure 22. Two views of the Cessna Citation III Model 650 wing geometry with aspect ratio 9.0 and leading-edge sweep of $27.2^{\circ}$. (a) Planform view; (b) isometric view. 
leading-edge sweep of $37^{\circ}$ before the break and $27^{\circ}$ after. The aspect ratio is 9.0 , and no wing dihedral is present. This wing is defined by three different airfoil sections at the root, break, and tip stations; and the twist distribution is incorporated in the airfoil coordinates. These three airfoils, along with the locations of the fixed and the three movable spline-support points are shown in Figure 23. Three movable points were chosen again based on the success of the Learjet design presented earlier.

In a private communication (Mr. Richard J. Crupper, Cessna Aircraft, Feb. 1984), the authors were told that the high-speed cruise conditions for the Citation III are Mach 0.81 at a lift coefficient of 0.21 . For the purposes of this study, a design was sought at Mach $=0.81$ and $C_{L}=0.57$ (for the initial configuration) to arbitrarily provide a more challenging example for TWING/QNM. The objective function to be used is again the wing drag-to-lift ratio.

The results of this design case are presented in Figures 24 through 28 at five span stations. Again, reasonably smooth pressure distributions and airfoil shapes are observed, with reduced shock strengths at every station (the $48.5 \%$ span station is nearly shockfree). The slight pressure peak at the root station at about $6 \%$ of chord might be eliminated by redistributing spline-support points and reinterpolating. The remaining stations are quite well behaved. Note that actually very little modification to the shape or thickness of any section was required to achieve the desired result. This is an indication that the wing was very well designed and 

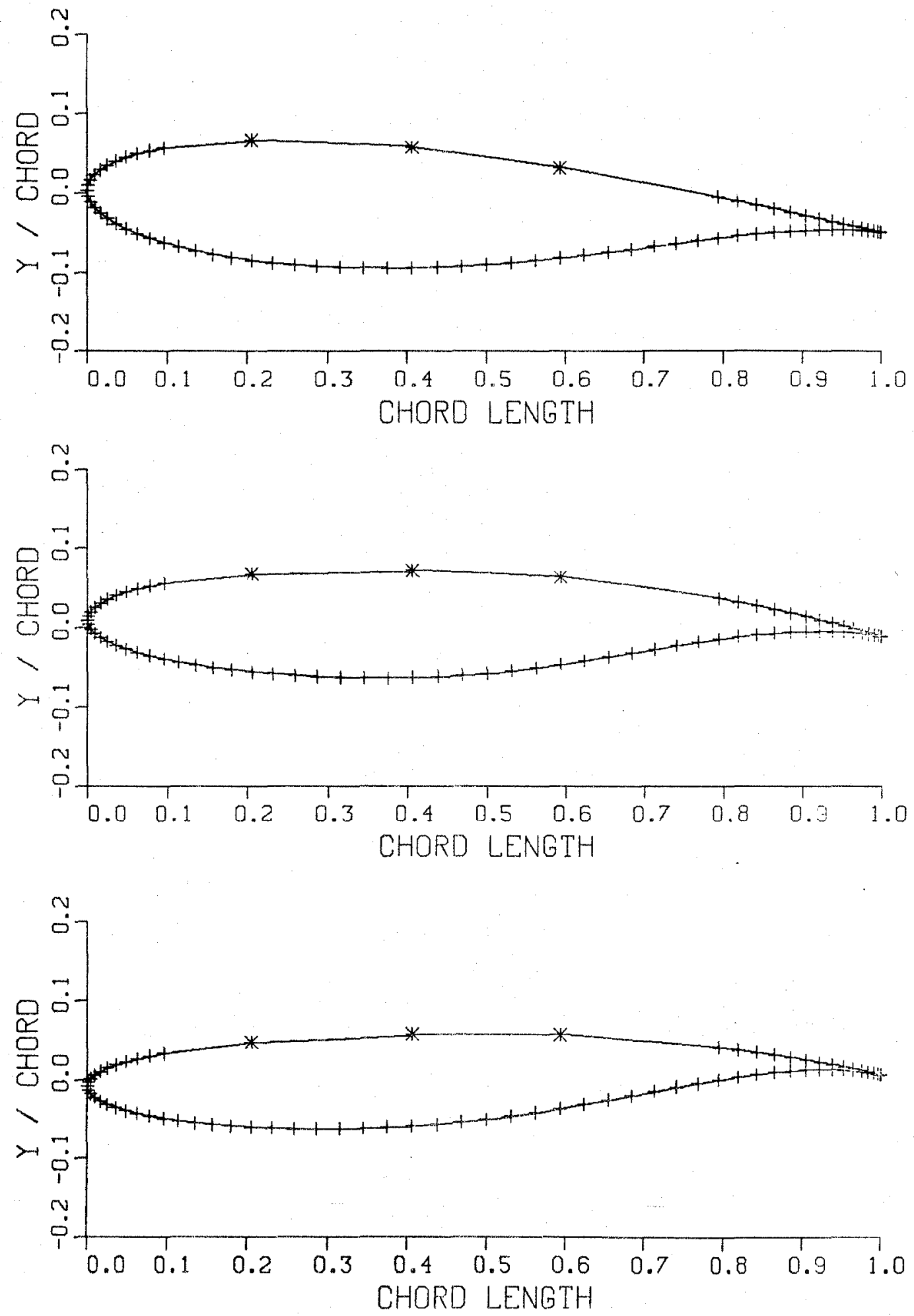

Figure 23. Location of the fixed $(t)$ and movable (*) spline-support points on the three Cessna airfoils.

(b) break station; (c) tip station.

(a) Root station; 


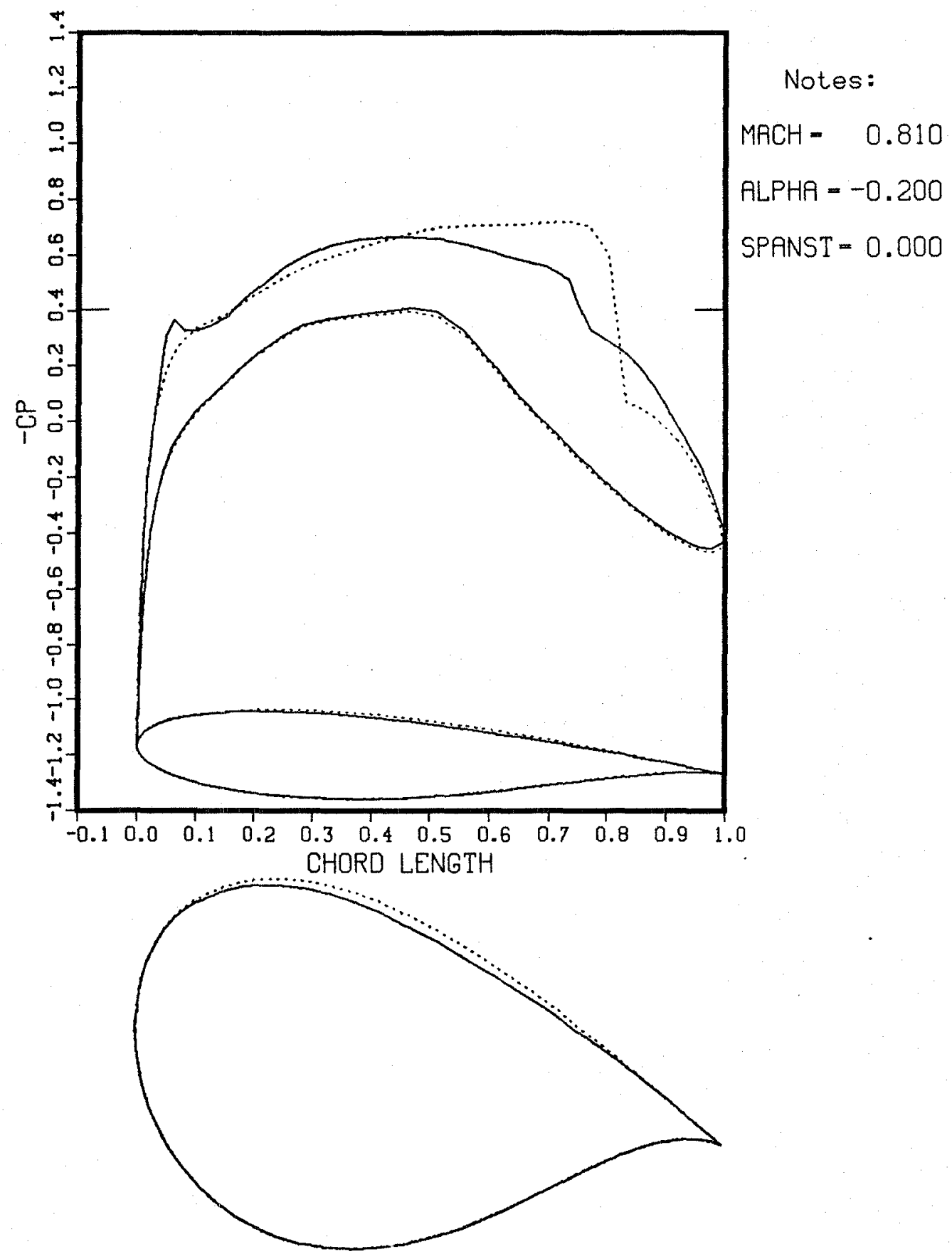

- AIRFOIL SHAPE AND PRESSURES AFTER OPTIMIZATION RUN ....... ORIGINAL CESSNA AIRFOIL AND PRESSURE DISTRIBUTION

Figure 24. TWING/QNM drag-to-lift ratio minimization result, Cessna Model 650 wing design: root span station. 


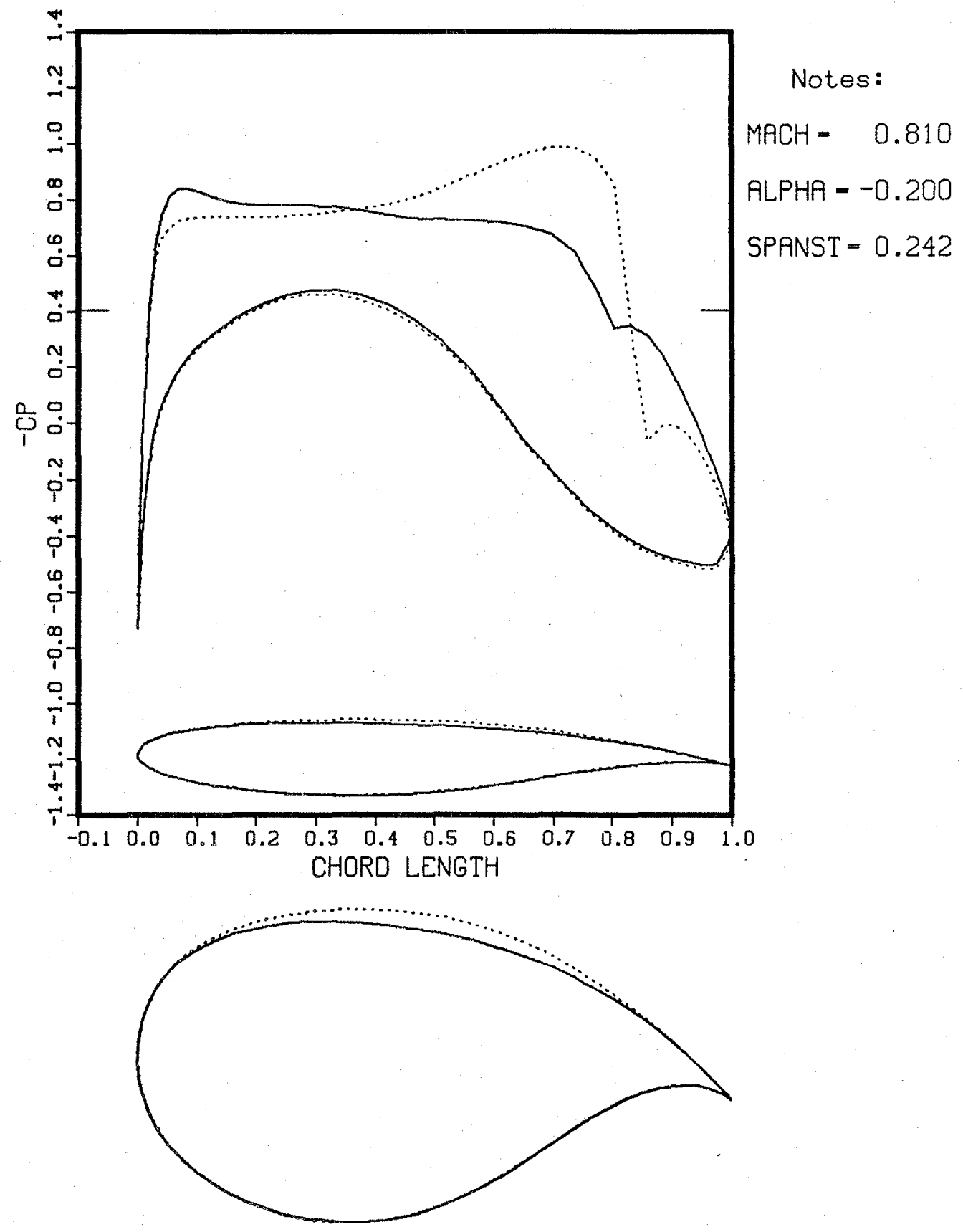

- AIRFOIL SHAPE AND PRESSURES AFTER OPTIMIZATION RUN ORIGINAL CESSNA AIRFOIL AND PRESSURE DISTRIBUTION

Figure 25. TWING/QNR: drag-to-1ift ratio minimization result, Cessna Mode1 650 wing design: $24.2 \%$ span station. 


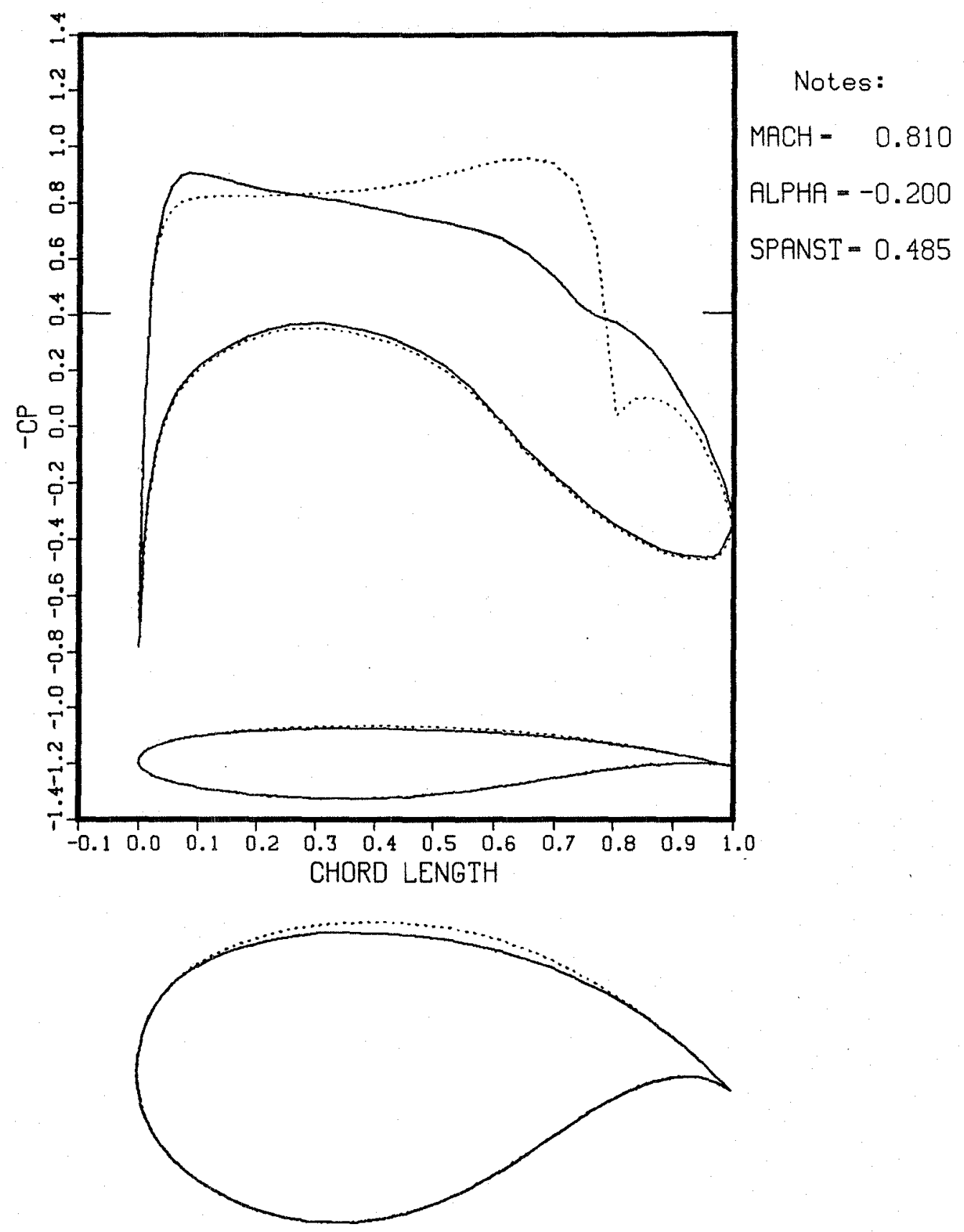

— AIRFOIL SHAPE AND PRESSURES AFTER OPTIMIZATION RUN ....... ORIGINAL CESSNA AIRFOIL AND PRESSURE DISTRIBUTION

Figure 26. TWING/QNM drag-to-lift ratio minimization result, Cessna Model 650 wing design: $48.5 \%$ span station. 


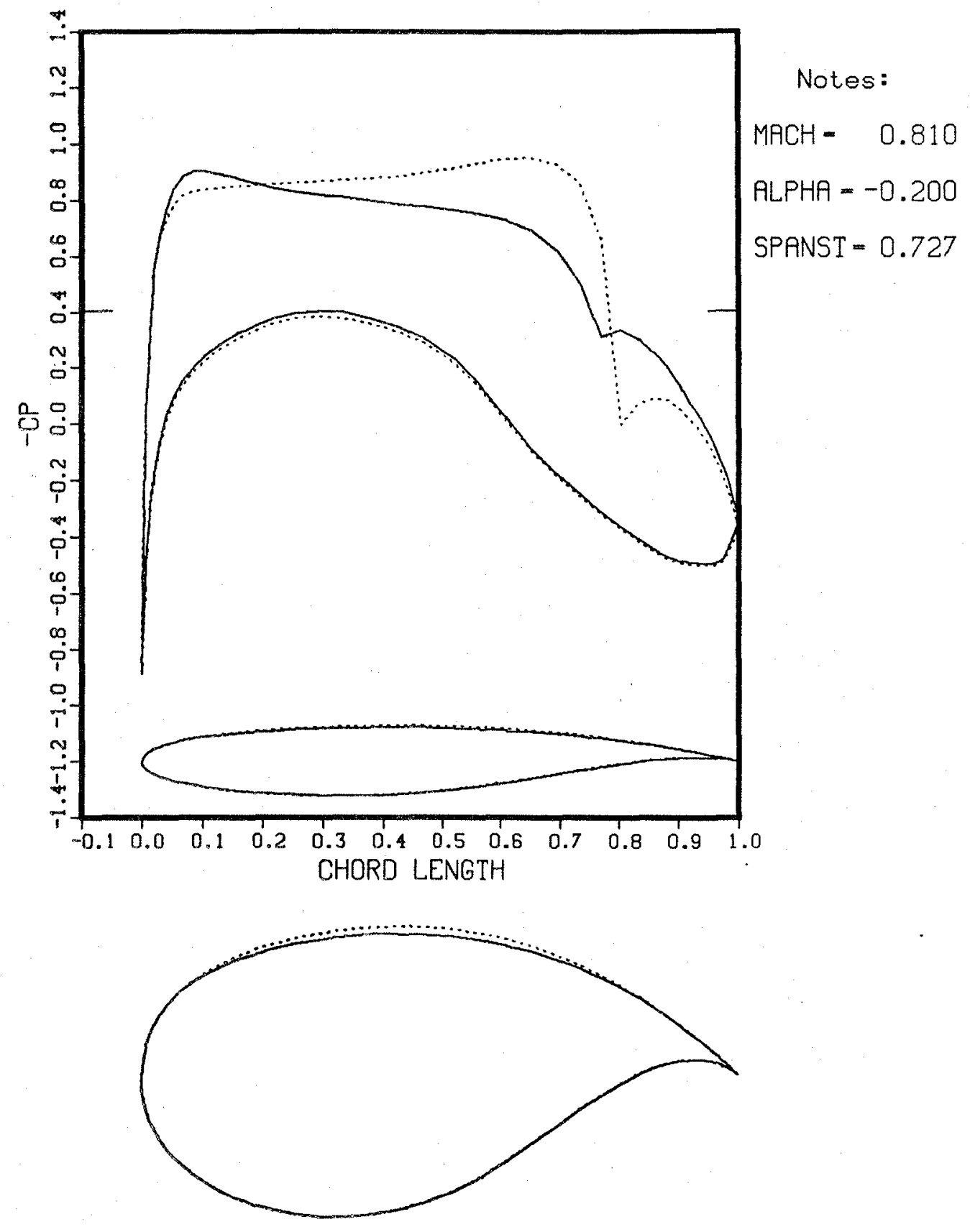

AIRFOIL SHAPE AND PRESSURES AFTER OPTIMIZATION RUN ....... ORIGINAL CESSNA AIRFOIL AND PRESSURE DISTRIBUTION

Figure 27. TWING/QNM drag-to-1ift ratio minimization result, Cessna Mode1 650 wing design: $72.7 \%$ span station. 

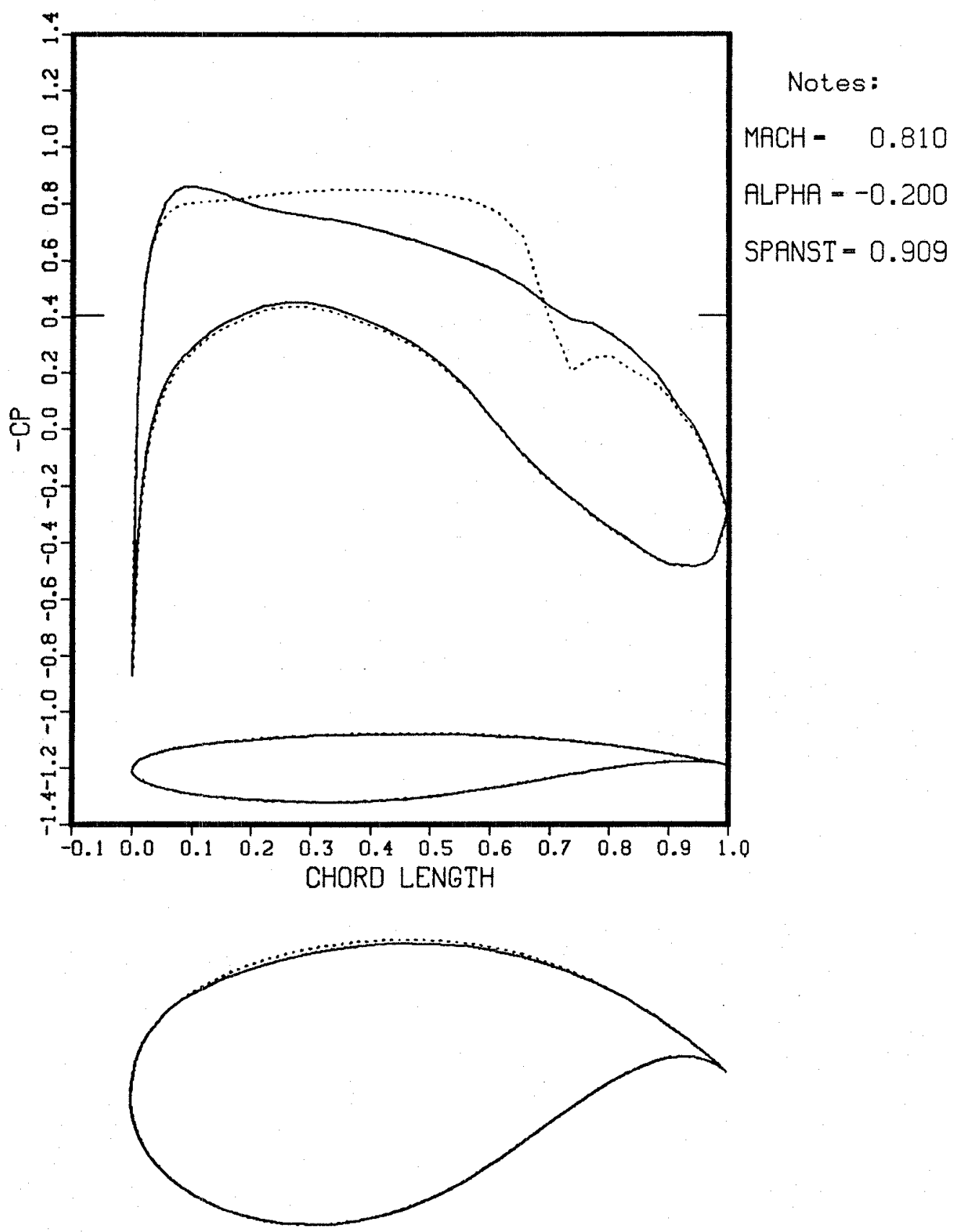

- AIRFOIL SHAPE AND PRESSURES AFTER OPTIMIZATION RUN …... ORIGINAL CESSNA AIRFOIL AND PRESSURE DISTRIBUTION

Figure 28. TWING/QNM drag-to-lift ratio minimization result, Cessna Mode1 650 wing design: $90.9 \%$ span station. 
highly efficient before any optimization redesign was carried out. Some data sumarizing this design are presented in Table 3.

Table 3. Summary of Cessna Model 650 Wing Design

\begin{tabular}{cccc}
\hline & Original wing & New wing & Percent change \\
\hline$C_{L}$ & 0.565 & 0.506 & -10.44 \\
$C_{D}$ & 0.00909 & 0.00438 & -51.82 \\
$L / D$ & 62.151 & 115.428 & +85.72 \\
\hline
\end{tabular}

The convergence history shown in Figure 29 is seen to display a behavior similar to that of the previous cases. This design required only six optimization iterations and was completed in just under 1 hr of Cray X-MP CPU time. The inviscid drag-to-lift ratio of this wing has been increased by over $85 \%$ at the expense of just over $10 \%$ of the lift, yielding a reasonable and efficient design at nominal computational expense. Note that the same procedure may be followed if a design with a higher lift is desired; the initial angle of attack would be set such that more lift is generated by the starting configuration. This should then compensate for the nominal amount of lift lost during the redesign process. The redefinition of the objective function such that decreases in lift below a specified minimum value would penalize the optimizer might be an alternative way of preserving lift. 


\title{
CHAPTER VI
}

\section{DISCUSSION AND CONCLUSIONS}

\begin{abstract}
A new tool has been developed to aid the aircraft designer concerned with efficient aircraft operation at transonic flight speeds. This was done by combining a sophisticated computational analysis program with an optimization algorithm using some new and promising techniques to provide the necessary interprogram communication. The availability of a supercomputer such as the Cray $\mathrm{X}-\mathrm{MP}$ and the high speed of the TWING analysis program have reduced by almost an order of magnitude the time required for a single threedimensional transonic flow-field solution. Because of this power, numerical optimization design procedures using such an analysis program should no longer be considered prohibitive because of cost. Indeed, exceptional designs have been shown to be achievable in less than $1 \mathrm{hr}$ of computer time.
\end{abstract}

The feasibility of utilizing the aerodynamic drag as computed by an inviscid potential-flow solver such as TWING has usually been discounted because of the expected low reliability and precision of such a computation. In a preliminary study for this work, the relative precision inherent in the computed drag (or, more 


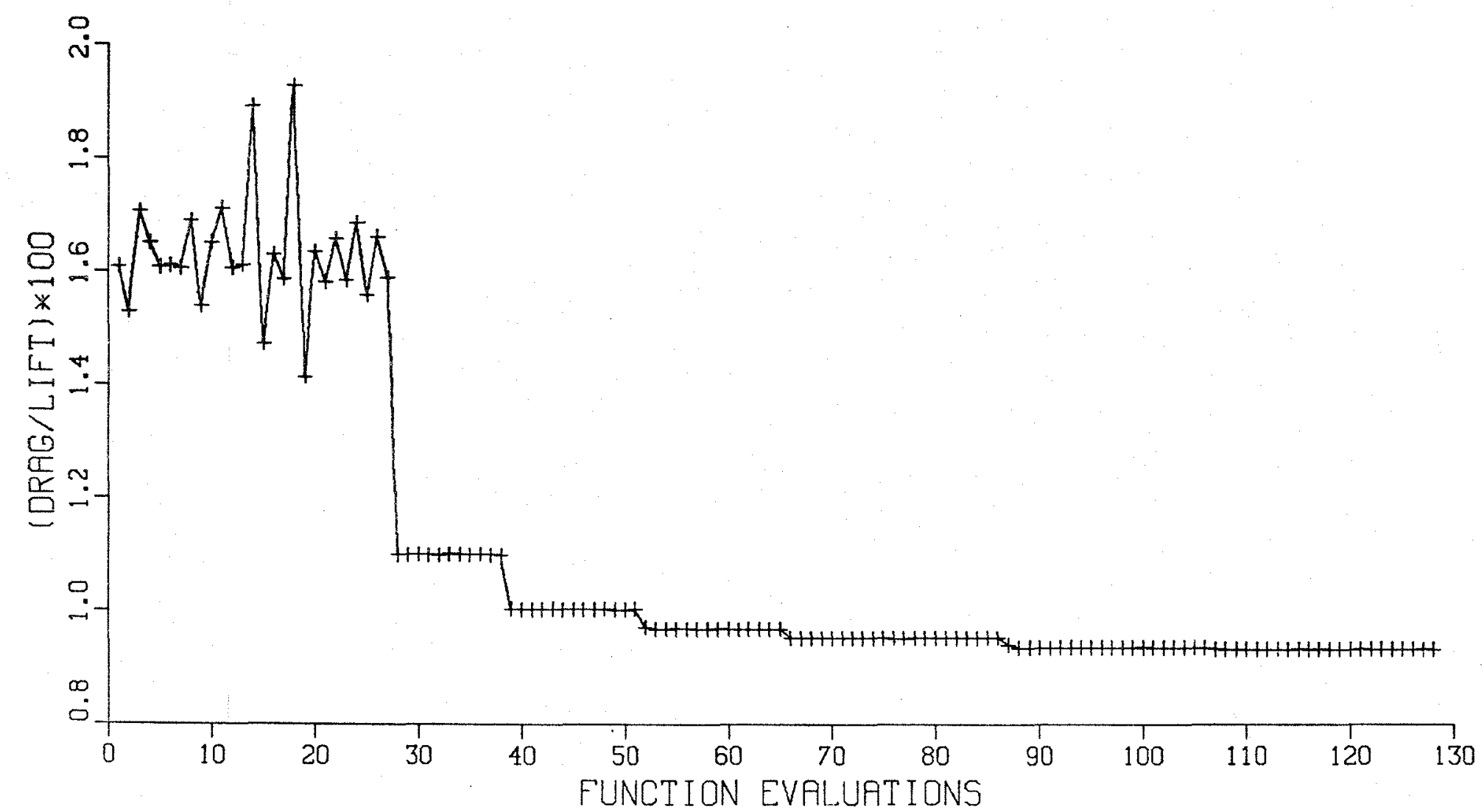

Figure 29. Objective function $\left(C_{D} / C_{L} \times 100\right)$ versus the number of TWING flow solutions: Cessna 650 wing. 
specifically, the drag-to-lift ratio) was found to be of nearly the same order as that of the more widely trusted $C_{p}$-objective value. This type of problem with the reliability of the objective is due to a lack of convergence of the flow-field solution. If tighter convergence levels are specified, the consistency, or repeatability, of the computation of the objective function is increased. Because of the speed of TWING, the potential solution may be converged to much more stringent levels than what must be accepted with a much slower program, and, consequently, the precision of the drag computation is correspondingly increased. This may well be the reason for the success of the designs presented here.

In addition to the question of available precision, the computed drag has also been thought to be misrepresentative in terms of its modeling of the physical situation. For some geometries and flow-field solutions this may be true; if so, it indicates the limitations of the problem formulation. Numerical truncation errors may also be a factor in computing drag. For these reasons, it should be stressed that the success of a particular design using drag as the objective function should not be judged in terms of the reduction of the objective value alone. Examination of the wing-surface shapes and the resulting pressure field by an experienced aerodynamicist will be necessary as the design progresses to be sure that physically reasonable modifications to the design are actually being performed. If this is not the case, a redistribution of the movable and fixed spline-support points was found to remedy the situation in the cases presented in this work. This was necessary for the C-141B 
only, not for the Learjet and Cessna. The feasibility of using the drag-to-1ift ratio as the design objective has been verified by the nearly shock-free design of the C-141B wing case presented, as there is virtually no chance of finding such a solution by simple luck (or by trial and error)!

The new SSPM method of geometrical perturbation reduced the required number of design variables significantly, and provided a sufficiently broad design space for many practical cases. The airfoil shapes defined at each design iteration are very reasonable, and in no case was a shape defined for which a flow solution could not be found. Because of the "implicit constraints" imposed on the design solution with this technique, formal constraints, which would retard the process or confuse the optimizer, are not required. Also, greater control over the acceptability of the final design is provided to the user by allowing only a specific portion of the airfoil surfaces to be modified. More specific flow problems may be attacked in this manner, without sacrificing other desirable airfoil characteristics.

The optimization routine QNMDIF has proved its "intelligence" quite convincingly based on the quality of the wing designs obtained, and the efficient and orderly inanner in which it progressed toward these solutions. Optimization divergence, or other general degradations to the design, were never observed. QNMDIF's performance was predictable and repeatable, at least when an adequate level of function precision was provided. This is directly related to the convergence level of the flow field solution, and a residual level of 
5.0E-7 yielded an objective function precision of 1 part in 10,000. This level was specified throughout this work, and was found to be adequate. Future investigations in which this precision level would be varied should yield some interesting facts about how the design is affected by the precision of the objective.

It is important to understand the limitations concerning the validity of a wing designed by this type of procedure. Since the optimization program received its information from an inviscid flow solution, it is obvious that such a design may only be valid when operating in a regime wherein viscous effects are unimportant. If in fact such an idealized flow solution can still provide the designer with important information, it should follow that a wing designed using such a solution should prove useful as well. By analyzing in a separate step the numerically designed wing configuration, any hysteresis effects in the course of the optimization design have been eliminated. Thus, the original and optimized wings are compared on the same basis. This is exactly what is displayed by the superimposed pressure distributions for each design case. An important conclusion from this discussion may now be stated: a wing designed using this type of computational procedure is valid only within the context of the assumptions inherent in the flow solutions; the design will be valid only where these assumptions are good approximations to the actual physical situation.

In summary, several important conclusions may be drawn from this research effort. These are stated as follows: 
1. Aerodynamic design by numerical optimization procedures need not be prohibitively expensive - the computational time that is required can be reduced greatly by employing advanced technology and supercomputer power.

2. By specifying tight convergence levels for the potential-flow solution, the numerical precision of the computed aerodynamic forces is sufficient to make possible their use as design objective functions.

3. Transonic wing designs obtained by such drag-to-liftratio minimization are quite practical, and yield physically reasonable pressure distributions.

4. Some user expertise and intervention is required; it will aid greatly in the production of useful designs.

5. Numerical optimization design procedures are reliable and very versatile - the extension to more complex wing-fuselage designs is possible and depends only on the availability of the flow-solution algorithm. Multiple design points may be specified, and a great deal of user control over the design is afforded.

The greatly enhanced efficiency of this numerical optimization design technique should broaden the range of its practical applications. This design method, in combination with more advanced flow simulations made possible by the use of the Euler or the full Navier-Stokes equations and more powerful computers, should allow the computation of truly realistic aircraft designs to become commonplace in the near future. 
1. Spreiter, J. R., "Transonic Aerodynamics - History and Statement of the Problem," from "Transonic Perspective Symposium," Vo1. 81 of Progress in Aeronautics and Astronautics, edited by D. Nixon, AIAA, New York, N.Y., 1982.

2. Lynch, F. T., "Commercial Transports - Aerodynamic Design for Cruise Performance Efficiency," from Transonic Perspective Symposium," Vo1. 81 of Progress in Aeronautics and Astronautics, edited by D. Nixon, AIAA, New York, N.Y., 1982.

3. Holst, T. L., Slooff, J. W., Yoshihara, H., and Ballhaus, W. F., Jr., "Applied Computational Transonic Aerodynamics," AGARDograph No. 266, AGARD AG-266, 1982.

4. Nieuwland, G. Y., The Computation by Lighthill's Method of Transonic Potential Flow around a Family of Quasi-Elliptical Aerofoils, NLR TR T.83, 1964.

5. Sobieczky, H., "Die Berechnung lokaler Raumlicher Uberschallfelder," ZAMM, 58T, 1978, pp. 215-216.

6. Sobieczky, H., Yu, N. J., Fung, K. Y., and Seebass, A. R., "New Method for Designing Shock-Free Transonic Configurations," AIAA Journa1, Vo1. 17, July 1979, pp. 722-729.

7. Nebeck, H. E. and Seebass, A. R., "Inviscid-Viscous Interaction in the Nearly Direct Design of Shock-Free Supercritical Airfoils," Computation of Viscous-Inviscid Interactions, AGARD CP-291, 1981.

8. Fung, K. Y., Seebass, A. R., Dickson, L. J., and Pearson, C. F., "An Effective Algorithm for Shock-Free Wing Design," AIAA Paper 81-1236, 1981.

9. Cosentino, G. B., "Modifying a General Aviation Airfoil for Supercritical Flight," AIAA Journal of Aircraft, Vo1. 20, No. 4, Apr. 1983, pp. 377-379.

10. Fung, K. Y., Sobieczky, H., and Seebass, R., "Shock-Free Wing Design," AIAA Paper.79-1557, 1979.

11. Raj, P., Miranda, L. R., and Seebass, A. R., "A Cost-Effective Method for Shock-Free Supercritical Wing Design," AIAA Paper 81-0383, 1981. 
12. Yu, N. J., "Efficient Transonic Shock-Free Wing Redesign Procedure Using a Fictitious Gas Method," AIAA Paper 79-0075, 1979.

13. Hicks, R. M., "Transonic Wing Design Using Potentia1-Flow Codes - Successes and Failures," SAE Technical Paper No. 810565, Apr. 1981.

14. Lores, M. E. and Hinson, B. L., "Transonic Design Using Computational Aerodynamics," from "Transonic Perspective Symposium," Vo1. 81 of Progress in Aeronautics and Astronautics, edited by D. Nixon, AIAA, New York, N.Y., 1982.

15. Hinson, M. L., "A Series of Airfoils Designed by Transonic Drag Minimization for Gates Learjet Aircraft," from "Transonic Perspective Symposium," Vo1. 81 of Progress in Aeronautics and Astronautics, edited by D. Nixon, AIAA, New York, N.Y., 1982.

16. Holst, T. L. and Thomas, S. D., "Numerical Solution of Transonic Wing Flowfields," AIAA Journal, Vo1. 21, No. 6, June 1983, pp. 863-870.

17. Holst, T. L., "Fast, Conservative Algorithm for Solving the Transonic Ful1-Potential Equation," AIAA Journal, Vo1. 18, Dec. 1980, pp. 1431-1439.

18. Thomas, S. D. and Holst, T. L., "Numerical Computation of Transonic Flow about Wing-Fuselage Configurations on a Vector Computer," AIAA Paper 83-0499, Jan. 1983.

19. Vanderplaats, G. N., "CONMIN - A FORTRAN Program for Constrained Function Minimization," NASA TM X-62,282, 1973.

20. Kennel1y, R. A., Jr., "Improved Method for Transonic Airfoil Design-by-Optimization," AIAA Paper 83-1864, July 1983.

21. Gi11, P. E., Murray, W., and Pitfield, R. A., "The Implementation of Two Revised Quasi-Newton Algorithms for Unconstrained Optimization," Report No. NAC 11, National Physical Laboratory, Division of Numerical Analysis and Computing, 1972.

22. Gill, P. E. and Murray, W., "Quasi-Newton Methods for Unconstrained Optimization," Journal of the Institute of Mathematics Applications, Vo1. 9, 1972, pp. 91-108.

23. Aidala, P. V., Davis, W. H., Jr., and Mason, W. H., "Smart Aerodynamic Optimization," AIAA Paper 83-1863, July 1983.

24. Sobieczky, H., "The Combination of a Geometry Generator with Transonic Design and Analysis Algorithms," AIAA Paper 83-1862, July 1983. 
25. Holst, T. L., "An Implicit Algorithm for the Conservative, Transonic Full Potential Equation Using an Arbitrary Mesh," AIAA Paper 78-1113, July 1978; also AIAA Journal, Vo1. 17, Oct. 1979, pp. 1038-1045.

26. Gill, P. E., Murray, W., and Wright, M. H., Practical Optimization, London, Academic Press, 1981.

27. McGhee, R. J., Beasley, W. D., and Somers, D. M., "Low-Speed Aerodynamic Characteristics of a $13 \%$ Thick Airfoil Section Designed for General Aviation Applications," NASA TM X-72697, 1975. 


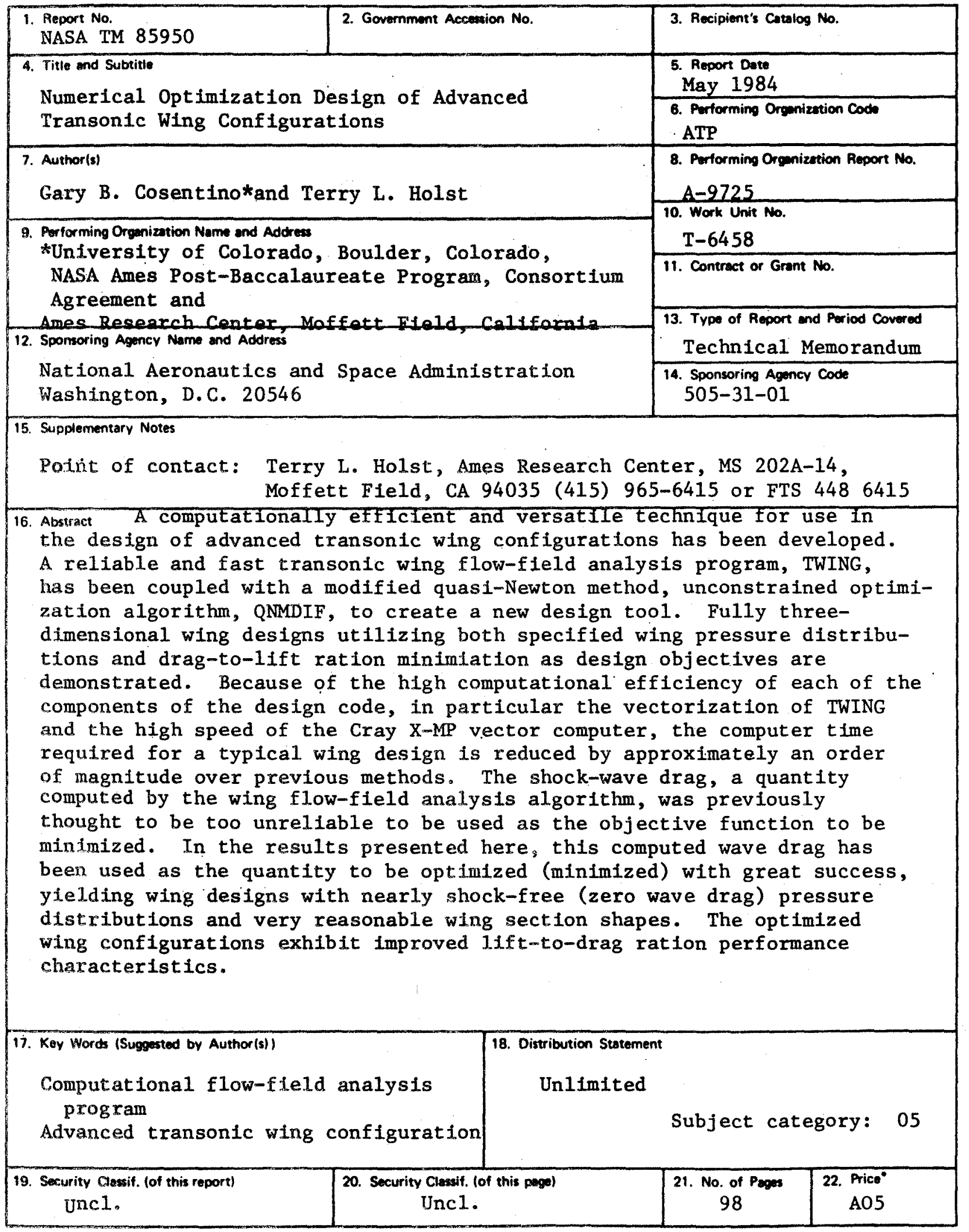

"For sale by the National Technicai Information Sorvice, Springfield, Virginia 22161 
End of Document 